COMMUNICATIONS IN

ANALYSIS AND GEOMETRY

Volume 7, Number 3, 451-495, 1999

\title{
Obstruction bundles, semiregularity, and Seiberg-Witten invariants
}

\author{
Robert Friedman AND John W. Morgan ${ }^{1}$
}

\section{Introduction.}

Recently, Seiberg and Witten have introduced new invariants for smooth 4-manifolds which have led to dramatic progress in understanding the $C^{\infty}$ properties of algebraic surfaces. Just as with Donaldson theory, the new invariants are computed from a moduli space which, in case the underlying 4-manifold is a Kähler surface $X$, can be identified with a moduli space of holomorphic objects. In Donaldson theory, the holomorphic moduli space is the space of holomorphic structures on a fixed $C^{\infty}$ complex vector bundle over $X$ satsifying an additional nondegeneracy condition, stability. Such moduli spaces have a rich geometric structure even for very simple Kähler surfaces, such as $\mathbb{P}^{2}$, and seem to become more progressively complicated as the surface becomes more complicated. In Seiberg-Witten theory, the relevant moduli spaces are the spaces of complex curves $D$ on $X$, which are thus parametrized by the Hilbert scheme of $X$, such that $D$ satisfies an additional numerical condition akin to stability. Now the structure of the Hilbert scheme of curves on a smooth surface is an interesting problem in algebraic geometry. However, it turns out for rather trivial reasons involving the Hodge index theorem that the geometric interest of the Seiberg-Witten moduli spaces of a surface $X$ is in a certain sense inversely proportional to the interest in $X$ itself as an abstract surface. Thus for example if $X$ is a minimal surface of general type the Seiberg-Witten moduli spaces are two reduced points corresponding to the trivial (empty) curve. Of course, it is this fact which enables one to prove that the first Chern class of the canonical bundle of a minimal surface of general type is a $C^{\infty}$ invariant up to sign. At the other extreme, if $X$ is a ruled surface over a curve $C$ of genus at least 2, then the Seiberg-Witten moduli spaces are connected with the

\footnotetext{
${ }^{1}$ The first author was partially supported by NSF grant DMS-92-03940. The second author was partially supported by NSF grant DMS-94-02988.
} 
Brill-Noether theory of special divisors on $C$, if $X=\mathbb{P}^{1} \times C$ is a product ruled surface, and to various interesting questions concerning stable bundles over $C$ in general. Our goal in this paper is to discuss these and other related examples.

The outline of this paper is as follows. In Section 1 we construct the Hilbert scheme of a complex surface via $\bar{\partial}$ methods. To our knowledge, such a construction has not appeared in the literature. In Section 2 we identify the deformation complex for the Seiberg-Witten equations of a Kähler surface in holomorphic terms and show that the Kuranishi model for the SeibergWitten equations is the same as the Kuranishi model for the equations defining the Hilbert scheme. In other words, the natural homeomorphism from the Seiberg-Witten moduli space to the Hilbert scheme of "stable" divisors on $X$ is an isomorphism of real analytic spaces. In Section 3 we discuss how to make computations in case the moduli space is smooth but does not have the expected dimension, using the Euler class of the obstruction bundle. These arguments and various generalizations are well-known to specialists in many different contexts. In Section 4 we apply this study to elliptic surfaces. There is a substantial overlap of the material in Sections 2-4 with the paper of Brussee [2].

The remainder of the paper is concerned with ruled surfaces. We discuss the infinitesimal and analytic structure of the moduli space for product ruled surfaces in Section 5, and then compute the invariant in the special case where the curve involved is a section of the surface (possibly with some fiber components). In Section 6, we deform the surface to a general ruled surface and show that the Hilbert scheme of sections is much better behaved: it is always smooth of the correct dimension. Using this result, we give another computation of the invariants in the case of a section. This computation goes back to Corrado Segre in 1889 [11] and was given a modern proof, for the case of the 0-dimensional invariant, by Ghione [6]. (Note that Segre considered the case of moduli spaces of sections of arbitrary dimension.) We shall give a quick description of these and related results. These methods generalize to compute the invariant in general homologically; the problem is that it is not known whether, for a general ruled surface, the Hilbert scheme always has the correct dimension. Finally we remark that the computation of the invariant is a special case of the transition formula for Seiberg-Witten invariants for 4-manifolds with $b_{2}^{+}=1$. This formula has been computed by the authors, by methods quite reminiscent of those in Section 6 , as well as by $\mathrm{Li}$ and Liu [9]. Thus our goal in Sections 5 and 6 has been, not so much to compute the invariant (although it is amusing to see the connections with the enumerative calculations of Brill-Noether theory) as it has been to see 
the relationship between the study of the Seiberg-Witten moduli spaces for ruled surfaces and questions in Brill-Noether theory as well as the theory of rank two stable bundles on curves.

\section{Structure of the Hilbert scheme.}

Let $X$ be an algebraic (or complex) surface, and let $D_{0}$ be an effective divisor on $X$. We do not assume that $D_{0}$ is smooth or even reduced. Let $H_{D_{0}, X}$ be the Hilbert scheme of all effective divisors $D$ on $X$ such that $c_{1}\left(\mathcal{O}_{X}(D)\right)=c_{1}\left(\mathcal{O}_{X}\left(D_{0}\right)\right)$ in $H^{2}(X ; \mathbb{Z})$. As a set, $H_{D_{0}, X}$ consists of all effective divisors $D$ homologous to $D_{0}$, i.e. algebraically equivalent to $D_{0}$. There is a morphism $H_{D_{0}, X} \rightarrow \operatorname{Pic} X$ whose fibers are projective spaces. Over $X \times H_{D_{0}, X}$ there is a tautological divisor $\mathcal{D}$ whose restriction to each slice $X \times\{t\}$ is the divisor $D_{t}$ on $X$ corresponding to $t$. A general reference for the construction of $H_{D_{0}, X}$ and its properties is [10].

The infinitesimal structure of $H_{D_{0}, X}$ is given as follows: from the natural exact sequence

$$
0 \rightarrow \mathcal{O}_{X} \rightarrow \mathcal{O}_{X}\left(D_{0}\right) \rightarrow \mathcal{O}_{D_{0}}\left(D_{0}\right) \rightarrow 0
$$

we have the associated long exact cohomology sequence. The Zariski tangent space to $H_{D_{0}, X}$ is naturally the space of sections of the normal bundle $H^{0}\left(D_{0} ; \mathcal{O}_{D_{0}}\left(D_{0}\right)\right)$. Note that the long exact cohomology sequence gives

$$
\begin{gathered}
0 \rightarrow H^{0}\left(X ; \mathcal{O}_{X}\left(D_{0}\right)\right) / H^{0}\left(X ; \mathcal{O}_{X}\right) \rightarrow H^{0}\left(D_{0} ; \mathcal{O}_{D_{0}}\left(D_{0}\right)\right) \\
\rightarrow H^{1}\left(X ; \mathcal{O}_{X}\right) \rightarrow H^{1}\left(X ; \mathcal{O}_{X}\left(D_{0}\right)\right) .
\end{gathered}
$$

Here $H^{0}\left(X ; \mathcal{O}_{X}\left(D_{0}\right)\right) / H^{0}\left(X ; \mathcal{O}_{X}\right)$ is the space of sections of $\mathcal{O}_{X}\left(D_{0}\right)$ modulo the line through a nonzero section vanishing along $D_{0}$, and is thus naturally the tangent space to the linear system $\left|D_{0}\right|$ at $D_{0}$. The map $H^{0}\left(D_{0} ; \mathcal{O}_{D_{0}}\left(D_{0}\right)\right) \rightarrow H^{1}\left(X ; \mathcal{O}_{X}\right)$ represents the infinitesimal change in the line bundle $\mathcal{O}_{X}\left(D_{0}\right)$. We let $K_{0}$ denote the image of $H^{0}\left(D_{0} ; \mathcal{O}_{D_{0}}\left(D_{0}\right)\right)$ in $H^{1}\left(X ; \mathcal{O}_{X}\right)$, so that $K_{0}$ is the kernel of the map from $H^{1}\left(X ; \mathcal{O}_{X}\right)$ to $H^{1}\left(X ; \mathcal{O}_{X}\left(D_{0}\right)\right)$ defined by $\sigma_{0}$. Thus there is an exact sequence

$$
0 \rightarrow H^{0}\left(X ; \mathcal{O}_{X}\left(D_{0}\right)\right) / H^{0}\left(X ; \mathcal{O}_{X}\right) \rightarrow H^{0}\left(D_{0} ; \mathcal{O}_{D_{0}}\left(D_{0}\right)\right) \rightarrow K_{0} \rightarrow 0 .
$$

The obstruction space to the deformation theory of $H_{D_{0}, X}$ is given as follows: let $K_{1}$ be the image of $H^{1}\left(X ; \mathcal{O}_{X}\left(D_{0}\right)\right)$ in $H^{1}\left(D_{0} ; \mathcal{O}_{D_{0}}\left(D_{0}\right)\right)$, or in other words the cokernel of the map from $H^{1}\left(X ; \mathcal{O}_{X}\right)$ to $H^{1}\left(X ; \mathcal{O}_{X}\left(D_{0}\right)\right)$ defined by $\sigma_{0}$. Then $K_{1}$ is the obstruction space to the functor corresponding 
to $H_{D_{0}, X}$. If $K_{1}=0$, then $H_{D_{0}, X}$ is scheme-theoretically smooth at $D_{0}$ of dimension equal to $\operatorname{dim} H^{0}\left(D_{0} ; \mathcal{O}_{D_{0}}\left(D_{0}\right)\right)$. (The converse is not necessarily true.) We say that $D_{0}$ is semiregular if $K_{1}=0$, or in other words if the map $H^{1}\left(X ; \mathcal{O}_{X}\left(D_{0}\right)\right) \rightarrow H^{0}\left(D_{0} ; \mathcal{O}_{D_{0}}\left(D_{0}\right)\right)$ is zero.

The following theorem was proved by Kodaira-Spencer [7] in the semiregular case (and was claimed by Severi):

Theorem 1.1. Let $D_{0}$ be a curve on $X$. Then the Zariski tangent space $T$ to $H_{D_{0}, X}$ at $D_{0}$ fits into an exact sequence

$$
0 \rightarrow H^{0}\left(X ; \mathcal{O}_{X}\left(D_{0}\right)\right) / H^{0}\left(X ; \mathcal{O}_{X}\right) \rightarrow T \rightarrow K_{0} \rightarrow 0,
$$

where $K_{0}=\operatorname{Ker}\left\{\times \sigma_{0}: H^{1}\left(X ; \mathcal{O}_{X}\right) \rightarrow H^{1}\left(X ; \mathcal{O}_{X}\left(D_{0}\right)\right)\right\}$. Locally analytically in a neighborhood of $D_{0}, H_{D_{0}, X}$ is defined by the vanishing of a convergent power series without constant or linear term from $T$ to

$$
K_{1}=\operatorname{Coker}\left\{\times \sigma_{0}: H^{1}\left(X ; \mathcal{O}_{X}\right) \rightarrow H^{1}\left(X ; \mathcal{O}_{X}\left(D_{0}\right)\right)\right\} .
$$

To prove Theorem 1.1, one can analyze the deformation theory and obstruction theory for $H_{D_{0}, X}$ via power series as in [7] and [10], and apply Schlessinger's theory. Here we give a $C^{\infty}$ proof of Theorem 1.1. Given $D_{0}$, let $L_{0}$ denote the $C^{\infty}$ complex line bundle defined by $\mathcal{O}_{X}\left(D_{0}\right)$. From this point of view, the scheme $H_{D_{0}, X}$ is the set (with real analytic structure) of all $C^{\infty}$ sections of $L_{0}$ which are complex analytic for some choice of holomorphic structure on $L_{0}$, modulo the action of the nowhere zero functions acting by multiplication. We fix a given holomorphic structure on $L_{0}$ with $\bar{\partial}$-operator simply denoted by $\bar{\partial}$, and we also fix a given nonzero holomorphic section $\sigma_{0}$ of $L_{0}$ for this holomorphic structure.

The equations which say that $s$ is a holomorphic section for some holomorphic structure on $L_{0}$ read as follows: there exist a $\bar{\partial}$-closed $(0,1)$-form $A$ such that $(\bar{\partial}+A)(s)=0$. Thus $H_{D_{0}, X}$ is the zero set of the function

$$
F_{0}: \operatorname{Ker} \bar{\partial} \oplus \Omega^{0}\left(L_{0}\right) \subset \Omega^{0,1}(X) \oplus \Omega^{0}\left(L_{0}\right) \rightarrow \Omega^{0,1}\left(L_{0}\right)
$$

defined by

$$
F_{0}(A, s)=(\bar{\partial}+A)(s)=\bar{\partial} s+A s,
$$

modulo the action of $\mathcal{G}_{\mathbb{C}}$, the complex gauge group, where $\mathcal{G}_{\mathbb{C}}$ is the multiplicative group of nowhere vanishing $C^{\infty}$ functions on $X$ and $\lambda \in \mathcal{G}_{\mathbb{C}}$ acts on $(A, s)$ via $(A-\bar{\partial} \lambda / \lambda, \lambda \cdot s)$. An easy calculation shows that $F_{0} \circ \lambda=\lambda F_{0}$. Of course, in order to analyze the equations, we need to pass to an appropriate 
Sobolev completion of all of these spaces, but we shall leave the details of this standard procedure to the reader.

Next we calculate the linearized complex. The space $\Omega^{0}\left(L_{0}\right)$ of all $C^{\infty}$ sections of $L_{0}$ is a vector space, and we may thus identify the tangent space to $\Omega^{0}\left(L_{0}\right)$ at a given section $\sigma_{0}$ vanishing at $D_{0}$ with $\Omega^{0}\left(L_{0}\right)$ again. The space of all $(0,1)$-conections on $L_{0}$ is an affine space over $\Omega^{0,1}(X)$, with origin the $\bar{\partial}$-operator corresponding to the given complex structure, and so its tangent space is $\Omega^{0,1}(X)$. The nowhere zero functions on $X$ may be (locally) identified with $\Omega^{0}(X)$, the set of all $C^{\infty}$ functions on $X$, via the exponential, and the differential at $s=\sigma_{0}, \lambda=0$ of

$$
\lambda \in \Omega^{0}(X) \mapsto e^{\lambda} \cdot s
$$

is multiplication by $\sigma_{0}: \lambda \mapsto \lambda \cdot \sigma_{0}$. Taking the differential of the $\mathcal{G}_{\mathbb{C}}$-action, we obtain a complex $\mathcal{C}_{0}$ :

$$
0 \rightarrow \Omega^{0}(X) \stackrel{d_{1}}{\longrightarrow} \operatorname{Ker} \bar{\partial} \oplus \Omega^{0}\left(L_{0}\right) \stackrel{d_{2}}{\longrightarrow} \Omega^{0,1}\left(L_{0}\right) .
$$

Here the map $d_{1}: \Omega^{0}(X) \rightarrow \operatorname{Ker} \bar{\partial} \oplus \Omega^{0}\left(L_{0}\right)$ sends $\lambda$ to $\left(-\bar{\partial} \lambda, \lambda \cdot \sigma_{0}\right)$ and $d_{2}$ : $\operatorname{Ker} \bar{\partial} \oplus \Omega^{0}\left(L_{0}\right) \rightarrow \Omega^{0,1}\left(L_{0}\right)$ sends $(A, s)$ to $\bar{\partial} s+A \sigma_{0}$. However, this complex is not elliptic. Thus, the restriction of $F_{1}$ to $\left(\operatorname{Im} d_{1}\right)^{\perp}$ is not Fredholm.

To remedy the above problem, consider instead the function $F(A, s)=$ $\pi \circ F_{0}(A, s)$, where $\pi: \Omega^{0,1}\left(L_{0}\right) \rightarrow \operatorname{Ker} \bar{\partial}$ is orthogonal projection onto the kernel of $\bar{\partial}$. (Note that $F$ is not in fact equivariant with respect to the action of $\mathcal{G}_{\mathbb{C}}$.)

Lemma 1.2. For $A$ in a neighborhood of zero in $\operatorname{Ker} \bar{\partial}, F(A, s)=0$ if and only if $F_{0}(A, s)=0$.

Proof. Clearly, if $F_{0}(A, s)=0$, then $F(A, s)=0$. Conversely, suppose that $F(A, s)=0$. This says that $F_{0}(A, s)$ is orthogonal to $\operatorname{Ker} \bar{\partial}$, so that $F_{0}(A, s)=(\bar{\partial}+A)(s)=\bar{\partial}^{*} \gamma$ for some $\gamma \in \Omega^{0,2}\left(L_{0}\right)$. By hypothesis $\bar{\partial} A=0$, so that $(\bar{\partial}+A)^{2}=0$. Applying $\bar{\partial}+A$ to $F_{0}$, we obtain $(\bar{\partial}+A) \bar{\partial}^{*} \gamma=0$, or in other words $\bar{\partial} \bar{\partial}^{*} \gamma+A \bar{\partial}^{*} \gamma=0$. We claim that, in this case, if $A$ lies in some neighborhood of zero, then $\bar{\partial}^{*} \gamma=0$ and thus $F_{0}(A, s)=0$. We may restrict $\gamma$ to $\left(\operatorname{Ker} \bar{\partial}^{*}\right)^{\perp}=\operatorname{Im} \bar{\partial}$, and in this case $\bar{\partial} \bar{\partial}^{*}$ is an isomorphism from $\operatorname{Im} \bar{\partial}$ to itself (after taking appropriate completions). Likewise, if $\pi_{0}$ denotes orthogonal projection from $\Omega^{0,2}\left(L_{0}\right)$ to $\operatorname{Im} \bar{\partial}$, then $\pi_{0} \circ\left(\bar{\partial} \bar{\partial}^{*}+A \bar{\partial}^{*}\right)$ is a bounded map from $\operatorname{Im} \bar{\partial}$ to itself, after taking appropriate completions, which is invertible for $A=0$ and so for $A$ in a neighborhood of zero. It 
follows that for $A$ in some neighborhood of zero, and for an arbitrary $\gamma \in$ $\Omega^{0,2}\left(L_{0}\right)$, if $\bar{\partial} \bar{\partial}^{*} \gamma+A \bar{\partial}^{*} \gamma=0$, then for $\gamma_{0}$ the projection of $\gamma$ to $\left(\operatorname{Ker} \bar{\partial}^{*}\right)^{\perp}$, we have $\bar{\partial}^{*} \gamma=\bar{\partial}^{*} \gamma_{0}$ and $\pi_{0} \circ\left(\bar{\partial} \bar{\partial}^{*}+A \bar{\partial}^{*}\right)\left(\gamma_{0}\right)=0$, so that $\gamma_{0}=0$ and $\gamma \in \operatorname{Ker} \bar{\partial}^{*}$. Hence $\bar{\partial}^{*} \gamma=0$ and so $F_{0}(A, s)=0$ as claimed.

The linearization of the equation $F$ and the gauge group action at $\left(0, \sigma_{0}\right)$ gives a complex $\mathcal{C}_{1}$ defined by the top line of the following commutative diagram:

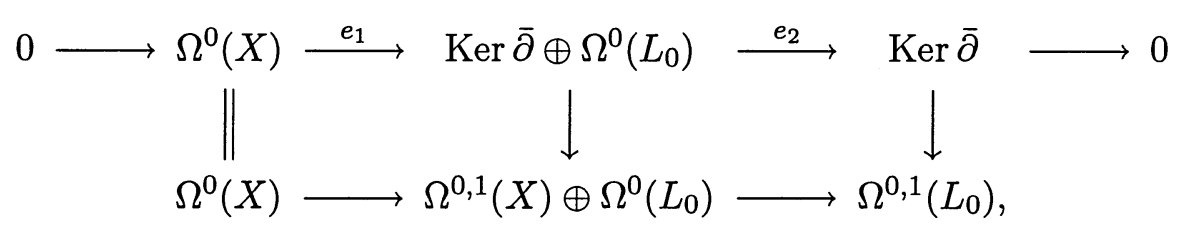

where the vertical maps are the natural inclusions, and the differentials are given by $e_{1}(\lambda)=\left(-\bar{\partial} \lambda, \lambda \sigma_{0}\right)$ and $e_{2}(A, s)=\bar{\partial} s+A \sigma_{0}$. There is a subcomplex $\mathcal{C}^{\prime}$ defined by

$$
\Omega^{0}\left(L_{0}\right) \stackrel{\bar{\partial}}{\rightarrow} \operatorname{Ker}\left\{\bar{\partial}: \Omega^{0,1}\left(L_{0}\right) \rightarrow \Omega^{0,2}\left(L_{0}\right)\right\}
$$

shifted up a dimension, with differential $\bar{\partial}$, and the quotient complex is isomorphic to the complex $\mathcal{C}^{\prime \prime}$ defined by:

$$
\Omega^{0}(X) \stackrel{\bar{\partial}}{\rightarrow} \operatorname{Ker}\left\{\bar{\partial}: \Omega^{0,1}(X) \rightarrow \Omega^{0,2}(X)\right\} .
$$

Thus the deformation complex is elliptic and so the restriction of $F$ to a slice for the $\mathcal{G}_{\mathbb{C}}$ action is Fredholm. Taking the long exact cohomology sequence associated to the exact sequence of complexes

$$
0 \rightarrow \mathcal{C}^{\prime} \rightarrow \mathcal{C}_{1} \rightarrow \mathcal{C}^{\prime \prime} \rightarrow 0
$$

we see that the cohomology of $\mathcal{C}_{1}$ fits into the exact sequence

$$
0 \rightarrow H^{0}\left(\mathcal{O}_{X}\right) \rightarrow H^{0}\left(L_{0}\right) \rightarrow H^{1}\left(\mathcal{C}_{1}\right) \rightarrow H^{1}\left(\mathcal{O}_{X}\right) \rightarrow H^{1}\left(L_{0}\right) \rightarrow H^{2}\left(\mathcal{C}_{1}\right) \rightarrow 0 .
$$

A routine calculation shows that the induced maps $H^{i}\left(\mathcal{O}_{X}\right) \rightarrow H^{i}\left(L_{0}\right)$ are given by multiplication by $\sigma_{0}$. Thus $H^{1}\left(\mathcal{C}_{1}\right)$ satisfies the exact sequence for $T$ given in Theorem 1.1 and $H^{2}\left(\mathcal{C}_{1}\right) \cong K_{1}$. This concludes the proof of Theorem 1.1.

The following identifies the quadratic term of the obstruction map: 
Proposition 1.3. Let $\xi \in H^{0}\left(\mathcal{O}_{D_{0}}\left(D_{0}\right)\right)$ be an element of the Zariski tangent space to $H_{D_{0}, X}$. Then the quadratic term in the obstruction map is equal to $\delta \xi \cup \xi \in H^{1}\left(\mathcal{O}_{D_{0}}\left(D_{0}\right)\right)$, where $\delta: H^{0}\left(\mathcal{O}_{D_{0}}\left(D_{0}\right)\right) \rightarrow H^{1}\left(\mathcal{O}_{X}\right)$ is the coboundary map in the natural long exact sequence.

Proof. It is easy to give the quadratic term of the obstruction map using the power series approach of [7]. In terms of the approach outlined here, the quadratic term of $F(A, s)$ is $\pi(A \cdot s)$, where $\bar{\partial} A=0$ and $A \sigma_{0}=-\bar{\partial} s$. If we identify the class of $(A, s)$ in $H^{1}\left(\mathcal{C}_{1}\right)$ with an element $\xi \in H^{0}\left(\mathcal{O}_{D_{0}}\left(D_{0}\right)\right)$, then it is easy to see that the class of $A$ in $H^{0,1}(X)=H^{1}\left(\mathcal{O}_{X}\right)$ is exactly $\delta \xi$. One then checks that the projection of $A \cdot s$ to $\operatorname{Ker} \bar{\partial}$ corresponds to $\delta \xi \cup \xi$.

Note that, if we apply $\delta: H^{1}\left(\mathcal{O}_{D_{0}}\left(D_{0}\right)\right) \rightarrow H^{2}\left(\mathcal{O}_{X}\right)$ to the element $\delta \xi \cup \xi$, we obtain $\delta \xi \cup \delta \xi \in H^{2}\left(\mathcal{O}_{X}\right)$, which is zero since $\delta \xi \cup \delta \xi=-\delta \xi \cup \delta \xi$, as $\mathcal{O}_{X}$ is a sheaf of commutative rings. Thus $\delta \xi \cup \xi$ lies in the image of $H^{1}\left(X ; \mathcal{O}_{X}\left(D_{0}\right)\right)$ in $H^{1}\left(D_{0} ; \mathcal{O}_{D_{0}}\left(D_{0}\right)\right)$.

There is also clearly a universal divisor $\mathcal{D} \subset X \times F^{-1}(0)$ defined by the vanishing of $s$. This completes the analytic construction of $H_{D_{0}, X}$ and the discussion of semiregularity. Note that we have not strictly speaking shown that $\mathcal{D}$ is a divisor on the complex space $X \times H_{D_{0}, X}$. This would need a discussion of relative $\bar{\partial}$-operators similar to, but easier than, the discussion in [4], Chapter IV, 4.2.3. In other words, we would need to show that $\mathcal{D}$ is a Cartier divisor in the possibly nonreduced complex space $X \times H_{D_{0}, X}$, which follows by showing that locally on $X \times H_{D_{0}, X}$, there is a holomorphic embedding of the complex space $X \times H_{D_{0}, X}$ in $X \times \mathbb{C}^{N}$ for some $N$ so that $\mathcal{D}$ is locally the restriction of a complex hypersurface. Finally, to identify this construction with the usual construction of $H_{D_{0}, X}$, and to make a geometric identification of $H_{D_{0}, X}$ possible, we would have to show that $H_{D_{0}, X}$ has a universal property. In other words, given a complex space $T$, not necessarily reduced, and a Cartier divisor on $X \times T$, flat over $T$, we need to exhibit a morphism of complex spaces from $T$ to $H_{D_{0}, X}$. This again can be done along the lines of [4], Chapter IV. In the cases described in this paper, $H_{D_{0}, X}$ will be smooth or a union of generically reduced components, and the arguments needed are substantially simpler than the arguments in the general case.

The divisor $\mathcal{D}$ is a Cartier divisor and so there is a holomorphic line bundle $\mathcal{O}_{X \times H_{D_{0}, X}}(\mathcal{D})$ over $X \times H_{D_{0}, X}$. Slant product with $c_{1}\left(\mathcal{O}_{X \times H_{D_{0}, X}}(\mathcal{D})\right)$ defines a map $H_{0}(X) \rightarrow H^{2}\left(H_{D_{0}, X}\right)$, and we let $\mu$ be the image of the natural generator of $H_{0}(X ; \mathbb{Z})$ under this map. Clearly $\mu=\pi_{2 *} c_{1}\left(\mathcal{O}_{X \times H_{D_{0}, X}}(\mathcal{D})\right)=$ 
$\pi_{2 *}[\mathcal{D}]$. For a fixed $p \in X$, there is the inclusion of the slice $\{p\} \times H_{D_{0}, X}$ in $X \times H_{D_{0}, X}$, and clearly $\mu$ is the first Chern class of the line bundle $\mathcal{O}_{X \times H_{D_{0}, X}}(\mathcal{D}) \mid\{p\} \times H_{D_{0}, X}$, under the natural identification of $\{p\} \times H_{D_{0}, X}$ with $H_{D_{0}, X}$. If $\mathcal{D}$ meets $\{p\} \times H_{D_{0}, X}$ properly, or in other words if there is no component $\mathcal{M}$ of $H_{D_{0}, X}$ such that $p$ lies in every divisor in $\mathcal{M}$, then $\mathcal{D} \cap\{p\} \times H_{D_{0}, X}$ is a Cartier divisor in $\{p\} \times H_{D_{0}, X} \cong H_{D_{0}, X}$, whose support is the set of divisors $D$ such that $p \in D$, and this divisor is a geometric representative for $\mu$. In fact, the divisor $\mu$ is an ample divisor on $H_{D_{0}, X}$, which can be shown for example by using the method of Chow schemes described in [10], Lecture 16, and identifying the numerical equivalence class of $\mu$ up to a positive rational multiple with the natural ample divisor on the Chow scheme.

There is another description of the complex line bundle corresponding to $\mu$. For $p \in X$, let $\mathcal{G}_{\mathbb{C}}^{0} \subset \mathcal{G}_{\mathbb{C}}$, the based gauge group, be the set of $\lambda \in \mathcal{G}_{\mathbb{C}}$ such that $\lambda(p)=1$. Thus the quotient of $\mathcal{G}_{\mathbb{C}}$ by $\mathcal{G}_{\mathbb{C}}^{0}$ is $\mathbb{C}^{*}$, and if instead of dividing out $F^{-1}(0)$ by the local action of $\mathcal{G}_{\mathbb{C}}$ we divide out by $\mathcal{G}_{\mathbb{C}}^{0}$, the result is a $\mathbb{C}^{*}$-bundle over $H_{D_{0}, X}$, which thus corresponds to a complex line bundle $\mathcal{L}_{0}(p)$. We claim that this line bundle has first Chern class equal to $\mu$. First note that there is a universal $C^{\infty}$ complex line bundle $\mathcal{L}_{0}$ over $X \times H_{D_{0}, X}$ whose restriction to the slice $\{p\} \times H_{D_{0}, X}$ is $\mathcal{L}_{0}(p)$. Here $\mathcal{L}_{0}$ is defined as follows: let $\mathcal{A}_{\mathbb{C}}^{*}\left(L_{0}\right)$ be the set of pairs $(A, s)$ where $A$ is a $(0,1)$-connection on $L_{0}$ and $s$ is a nonzero section of $L_{0}$. Then $\mathcal{G}_{\mathbb{C}}$ acts freely on $\mathcal{A}_{\mathbb{C}}^{*}\left(L_{0}\right)$; let the quotient be denoted $\mathcal{B}_{\mathbb{C}}\left(L_{0}\right)$. Since $\mathcal{G}_{\mathbb{C}}$ also acts as a group of automorphisms of $L_{0}$, there is a line bundle $\mathcal{L}_{0}$ over $X \times \mathcal{B}_{\mathbb{C}}\left(L_{0}\right)$ obtained by dividing out $L_{0} \times \mathcal{A}_{\mathbb{C}}^{*}\left(L_{0}\right)$ by the action of $\mathcal{G}_{\mathbb{C}}$. We also denote the restriction of this line bundle to $X \times F^{-1}(0)$ by $\mathcal{L}_{0}$. So we must identify $\mathcal{L}_{0}$ with $c_{1}\left(\mathcal{O}_{X \times H_{D_{0}, X}}(\mathcal{D})\right)$ (at least on the reduction of $\left.H_{D_{0}, X}\right)$. The point is that the tautological section $(s,(A, s))$ of the pullback of $L_{0}$ to $X \times \mathcal{A}_{\mathbb{C}}^{*}\left(L_{0}\right)$ is $\mathcal{G}_{\mathbb{C}}$-equivariant and so descends to a section of $\mathcal{L}_{0}$ over $X \times \mathcal{B}_{\mathbb{C}}\left(L_{0}\right)$. Restricting to $X \times F^{-1}(0)$, we see that $\mathcal{L}_{0}$ has a section vanishing at $\mathcal{D}$, and this identifies $\mathcal{L}_{0}$ with $\mathcal{O}_{X \times H_{D_{0}, X}}(\mathcal{D})$.

\section{Deformation theory for Seiberg-Witten moduli spaces of Kähler surfaces.}

In this section we recall the description of Seiberg-Witten moduli spaces for Kähler surfaces and compare this description to the discussion of the Hilbert scheme in the previous section. For general references on SeibergWitten moduli spaces of Kähler surfaces, see [12], [3], as well as [5]. 
For a given metric $g$ on $X$ and $\operatorname{Spin}^{c}$ structure $\xi$ on $X$ with determinant $L$, the (unperturbed) Seiberg-Witten equations for a pair $(A, \psi)$, where $A$ is a connection on $L$ and $\psi$ is a section of $\mathbb{S}^{+}(\xi)$, the plus spinor bundle associated to $\xi$, are

$$
\begin{aligned}
\not_{A} \psi & =0 \\
F_{A}^{+}=q(\psi) & =\psi \otimes \psi^{*}-\frac{|\psi|^{2}}{2} \mathrm{Id} .
\end{aligned}
$$

We let $\mathcal{M}_{g}(\xi)$ be the corresponding moduli space. In case $X$ is a Kähler surface and $g$ is a Kähler metric with associated Kähler form $\omega, \mathbb{S}^{+}(\xi) \cong$ $\Omega^{0}\left(L_{0}\right) \oplus \Omega^{0,2}\left(L_{0}\right)$ for a complex line bundle $L_{0}$, and $\mathbb{S}^{-}(\xi) \cong \Omega^{0,1}\left(L_{0}\right)$. Moreover $L_{0}^{\otimes 2}=L \otimes K_{X}$, where $L=\operatorname{det} \xi$. We assume that $\omega \cdot c_{1}(L) \neq 0$, so that there are no reducible solutions, and for simplicity we fix $\omega \cdot c_{1}(L)<0$. In this case, writing $\psi$ in components $(\alpha, \beta)$, where $\alpha$ is a section of $\Omega^{0}\left(L_{0}\right)$ and $\beta$ is a section of $\Omega^{0,2}\left(L_{0}\right)$, the Seiberg-Witten equations become

$$
\begin{aligned}
F^{0,2} & =\bar{\partial} A^{0,1}=\bar{\alpha} \beta ; \\
\left(F_{A}^{+}\right)^{1,1} & =\frac{i}{2}\left(|\alpha|^{2}-|\beta|^{2}\right) \omega ; \\
\bar{\partial}_{A} \alpha+\bar{\partial}_{A}^{*} \beta & =0 .
\end{aligned}
$$

(Here the metric $g$ defines a Hermitian metric on $L_{0}$ and thus a conjugate linear isomorphism $\Omega^{0}\left(L_{0}\right) \rightarrow \Omega^{0}\left(L_{0}^{*}\right)$, and $\bar{\alpha}$ denotes the image of $\alpha$ under this isomorphism.) Under the assumption that $\alpha \neq 0$, the equations $\bar{\partial} A^{0,1}=$ $\bar{\alpha} \beta$ and $\bar{\partial}_{A}\left(\bar{\partial}_{A} \alpha+\bar{\partial}_{A}^{*} \beta\right)=0$ imply that $\beta=0$, and that $A$ is a $(1,1)$-conection on $L[12,3,5]$. Hence $L$ and $L_{0}$ have given holomorphic structures, and $\bar{\partial}_{A} \alpha=0$, so that $\alpha$ is a nonzero holomorphic section of $L_{0}$. Thus $\alpha$ defines an effective divisor $D$ with $L_{0}=\mathcal{O}_{X}(D)$. Taking gauge equivalence defines $\alpha$ up to scalars, or in other words as an element of $|D|$. Thus to each element of $\mathcal{M}_{g}(\xi)$, there is a well-defined element of $H_{D_{0}, X}$ for some fixed divisor $D_{0}$ such that the $C^{\infty}$ line bundle underlying $\mathcal{O}_{X}\left(2 D_{0}\right) \otimes K_{X}^{-1}$ is $L$. Conversely, to every point of $H_{D_{0}, X}$ we can associate an irreducible solution of the SeibergWitten equations mod gauge equivalence, in other words a point of $\mathcal{M}_{g}(\xi)$, which essentially follows from a theorem of Kazdan-Warner. It is easy to see that the map from $\mathcal{M}_{g}(\xi)$ to $H_{D_{0}, X}$ is a homeomorphism, and we shall show that it is an isomorphism of real analytic spaces in a suitable sense. As in the previous section, we shall pass to Sobolev completions of all of the spaces of $C^{\infty}$ sections involved without making the choice of completions explicit. 
We begin by discussing the deformation complex associated to the Seiberg-Witten equations for a Kähler surface. For a general Riemannian. 4-manifold $X$, at an irreducible solution $\left(A_{0}, \psi\right)$ to the Seiberg-Witten equations, the appropriate deformation complex $\mathcal{C}$ is

$$
0 \rightarrow i \Omega^{0}(X ; \mathbb{R}) \stackrel{\delta_{1}}{\longrightarrow} i \Omega^{1}(X ; \mathbb{R}) \oplus \mathbb{S}^{+}(\xi) \stackrel{\delta_{2}}{\longrightarrow} i \Omega_{+}^{2}(X ; \mathbb{R}) \oplus \mathbb{S}^{-}(\xi) \rightarrow 0 .
$$

Here $\Omega_{+}^{2}(X)$ is the space of $C^{\infty}$ self-dual 2-forms. The differentials are as follows: $\delta_{1}(\lambda)=(-2 d \lambda, \lambda \psi)$ and

$$
\delta_{2}(A, \eta)=\left(d^{+} A-D q_{\psi}(\eta), \not \partial \eta+\frac{1}{2} A \cdot \psi\right) .
$$

Here $d^{+}$is the self-dual part of $d, D q_{\psi}$ is the differential of the quadratic map $q$ in the SW equations, evaluated at $\psi$ on $\psi$, and $\frac{1}{2} A \cdot \psi$ is the linear term of $\not_{A+A_{0}} \psi$. A calculation shows that

$$
D q_{\psi}(\eta)=\eta \otimes \psi^{*}+\psi \otimes \eta^{*}-\operatorname{Re}\langle\psi, \eta\rangle \mathrm{Id} .
$$

In general, it seems to be somewhat difficult to analyze this complex. In the case of a Kähler surface $X$, however, we can give a very explicit description of the cohomology of the deformation complex. First we recall the notation of the previous section:

$$
\begin{aligned}
& K_{0}=\operatorname{Ker}\left\{\times \sigma_{0}: H^{1}\left(\mathcal{O}_{X}\right) \rightarrow H^{1}\left(L_{0}\right)\right\} ; \\
& K_{1}=\operatorname{Coker}\left\{\times \sigma_{0}: H^{1}\left(\mathcal{O}_{X}\right) \rightarrow H^{1}\left(L_{0}\right)\right\} ; \\
& K_{2}=\operatorname{Ker}\left\{\times \sigma_{0}: H^{2}\left(\mathcal{O}_{X}\right) \rightarrow H^{2}\left(L_{0}\right)\right\} .
\end{aligned}
$$

(Note that $\times \sigma_{0}: H^{2}\left(\mathcal{O}_{X}\right) \rightarrow H^{2}\left(L_{0}\right)$ is surjective.)

Theorem 2.1. Suppose that $X$ is a Kähler surface with Kähler metric $g$ and associated Kähler form $\omega$. Let $\sigma_{0}$ be a nonzero holomorphic section of $L_{0}$ corresponding to an irreducible solution $\left(\sigma_{0}, 0\right)$ of the Seiberg-Witten equations. Then the Zariski tangent space $H^{1}(\mathcal{C})$ to the Seiberg-Witten moduli space sits in an exact sequence

$$
0 \rightarrow H^{0}\left(L_{0}\right) / \mathbb{C} \sigma_{0} \rightarrow H^{1}(\mathcal{C}) \rightarrow K_{0} \rightarrow 0
$$

and the obstruction space $H^{2}(\mathcal{C})$ sits in an exact sequence

$$
0 \rightarrow K_{1} \rightarrow H^{2}(\mathcal{C}) \rightarrow K_{2} \rightarrow 0 .
$$


Obstruction bundles, semiregularity, and Seiberg-Witten invariants 461

Proof. The complex $\mathcal{C}$ has the following complex as its symbol complex:

$$
0 \rightarrow i \Omega^{0}(X ; \mathbb{R}) \stackrel{(d, 0)}{\longrightarrow} i \Omega^{1}(X ; \mathbb{R}) \oplus \mathbb{S}^{+}(\xi) \stackrel{\left(d^{+}, \phi\right)}{\longrightarrow} i \Omega_{+}^{2}(X ; \mathbb{R}) \oplus \mathbb{S}^{-}(\xi) \rightarrow 0 .
$$

Thus it is elliptic and its (real) index is the same as the index of the above complex, namely

$$
1-b_{1}(X)+b_{2}^{+}(X)-2\left(h^{0}\left(L_{0}\right)+h^{2}\left(L_{0}\right)-h^{1}\left(L_{0}\right)\right)=2 \chi\left(\mathcal{O}_{X}\right)-2 \chi\left(L_{0}\right) .
$$

Here we have used the identification of $\not \partial$ with $\bar{\partial}+\bar{\partial}^{*}$ up to a factor of $\sqrt{2}$. Note that $\delta_{1}(\lambda)=0$ if and only if $\lambda$ is constant and $\lambda \sigma_{0}=0$. Thus $H^{0}(\mathcal{C})=0$, which just says that the point $\left(A,\left(\sigma_{0}, 0\right)\right.$ is an irreducible solution to the Seiberg-Witten equations, and we must identify the terms $H^{1}(\mathcal{C})$ and $H^{2}(\mathcal{C})$. Identify $i \Omega^{1}(X ; \mathbb{R})$ with $\Omega^{0,1}(X), \mathbb{S}^{+}(\xi)$ with $\Omega^{0}\left(L_{0}\right) \oplus \Omega^{0,2}\left(L_{0}\right)$, $i \Omega_{+}^{2}(X ; \mathbb{R})$ with $i \Omega^{0}(X ; \mathbb{R}) \omega \oplus \Omega^{0,2}(X)$, and $\mathbb{S}^{-}(\xi)$ with $\Omega^{0,1}\left(L_{0}\right)$. Under these identifications, for $\lambda \in i \Omega^{0}(X ; \mathbb{R}), \delta_{1} \lambda=\left(-\bar{\partial} \lambda, \lambda \sigma_{0}, 0\right)$ which has image inside $\Omega^{0,1}(X) \oplus \Omega^{0}\left(L_{0}\right)$. Moreover, $\delta_{2}\left(A^{0,1}, \alpha, \beta\right)$ is given by

$$
\left(\left(\bar{\partial} \bar{A}^{0,1}+\partial A^{0,1}\right)^{+}-\operatorname{Re}\left\langle\sigma_{0}, \alpha\right\rangle i \omega, \bar{\partial} A^{0,1}-\bar{\sigma}_{0} \beta, \bar{\partial} \alpha+\bar{\partial}^{*} \beta+\frac{1}{2} A^{0,1} \sigma_{0}\right) .
$$

After identifying $i \Omega^{0}(X ; \mathbb{R}) \omega$ with $\Omega^{0}(X ; \mathbb{R})$ by taking $-i$ times the contraction $\Lambda$ with $\omega$, we can write this as

$$
\delta_{2}\left(A^{0,1}, \alpha, \beta\right)=\left(T_{1}\left(A^{0,1}, \alpha\right), T_{2}\left(A^{0,1}, \alpha\right)+S(\beta)\right),
$$

where (since $\omega \wedge \omega$ is twice the volume form)

$$
\begin{aligned}
T_{1}\left(A^{0,1}, \alpha\right) & =-i\left(\Lambda \bar{\partial} \bar{A}^{0,1}+\Lambda \partial A^{0,1}\right)-2 \operatorname{Re}\left\langle\sigma_{0}, \alpha\right\rangle \in \Omega^{0}(X ; \mathbb{R}) \\
T_{2}\left(A^{0,1}, \alpha\right) & =\left(\bar{\partial} A^{0,1}, \bar{\partial} \alpha+\frac{1}{2} A^{0,1} \sigma_{0}\right) \in \Omega^{0,2}(X) \oplus \Omega^{0,1}\left(L_{0}\right) ; \\
S(\beta) & =\left(-\bar{\sigma}_{0} \beta, \bar{\partial}^{*} \beta\right) \in \Omega^{0,2}(X) \oplus \Omega^{0,1}\left(L_{0}\right) .
\end{aligned}
$$

Thus $\delta_{2}\left(A^{0,1}, \alpha, \beta\right)=0$ if and only if $T_{1}\left(A^{0,1}, \alpha\right)=0$ and $T_{2}\left(A^{0,1}, \alpha\right)+S(\beta)=$ 0 . Next we claim:

Lemma 2.2. $T_{2}\left(A^{0,1}, \alpha\right)+S(\beta)=0$ if and only if $\beta=0$ and $T_{2}\left(A^{0,1}, \alpha\right)=$ 0 . Moreover, $\bar{\partial} A^{0,1}=\bar{\sigma}_{0} \beta$ and $\bar{\partial}\left(\bar{\partial} \alpha+\bar{\partial}^{*} \beta+\frac{1}{2} A^{0,1} \sigma_{0}\right)=0$ if and only if $\beta=0$ and $\bar{\partial} A^{0,1}=0$. 
Proof. Clearly, if $\beta=0$ and $T_{2}\left(A^{0,1}, \alpha\right)=0$, then $T_{2}\left(A^{0,1}, \alpha\right)+S(\beta)=0$. Conversely, suppose that $T_{2}\left(A^{0,1}, \alpha\right)+S(\beta)=0$, or in other words that $\bar{\partial} A^{0,1}=\bar{\sigma}_{0} \beta$ and $\bar{\partial} \alpha+\bar{\partial}^{*} \beta+\frac{1}{2} A^{0,1} \sigma_{0}=0$. Taking $\bar{\partial}$ of the second equation, we find that

$$
\bar{\partial} A^{0,1} \cdot \sigma_{0}+\bar{\partial} \bar{\partial}^{*} \beta=\left|\sigma_{0}\right|^{2} \beta+\bar{\partial} \bar{\partial}^{*} \beta=0 .
$$

Taking the inner product with $\beta$ shows that

$$
\int_{X}\left|\sigma_{0}\right|^{2}|\beta|^{2}+\left\|\bar{\partial}^{*} \beta\right\|^{2}=0 \text {. }
$$

Hence $\beta=0$, and clearly then $T_{2}\left(A^{0,1}, \alpha\right)=0$ as well. The proof of the second assertion is similar.

Now we exhibit an isomorphism from $H^{1}(\mathcal{C})$ to $H^{1}\left(\mathcal{C}_{1}\right)$, where $\mathcal{C}_{1}$ is the complex defined in the previous section, up to a factor of 2 (which arises because in our point of view $A^{0,1}$ is a connection on $L_{0}^{\otimes 2} \otimes K_{X}$ rather than on $\left.L_{0}\right): H^{1}\left(\mathcal{C}_{1}\right)$ is the quotient of

$$
\left\{\left(A^{0,1}, \alpha\right): \bar{\partial} A^{0,1}=0, \bar{\partial} \alpha+\frac{1}{2} A^{0,1} \sigma_{0}=0\right\}
$$

by the subgroup of elements of the form $\left(-2 \bar{\partial} f, f \sigma_{0}\right)$, where $f$ is a complex valued $C^{\infty}$ function. To exhibit this isomorphism, given a class in $H^{1}(\mathcal{C})$ represented by $\left(A^{0,1}, \alpha, \beta\right)$, then, by Lemma $2.2, \beta=0$ and $\bar{\partial} \alpha+\frac{1}{2} A^{0,1} \sigma_{0}=0$. Moreover $\left(A^{0,1}, \alpha\right)$ is well-defined up to the subgroup of the form $\left(-2 \bar{\partial} \lambda, \lambda \sigma_{0}\right)$, where $\lambda$ is a purely imaginary $C^{\infty}$ function. Thus $\left(A^{0,1}, \alpha\right)$ defines an element of $H^{1}\left(\mathcal{C}_{1}\right)$.

Conversely, start with a representative $\left(A^{0,1}, \alpha\right)$ for an element of $H^{1}\left(\mathcal{C}_{1}\right)$. Then $\left(A^{0,1}, \alpha, 0\right)$ satisfies $T_{2}\left(A^{0,1}, \alpha\right)=0$ but not necessarily $T_{1}\left(A^{0,1}, \alpha\right)=0$. On the other hand, we can change $\left(A^{0,1}, \alpha\right)$ by an element of the form $\left(-2 \bar{\partial} h, h \sigma_{0}\right)$, where $h$ is a real $C^{\infty}$ function, without affecting $T_{2}\left(A^{0,1}, \alpha\right)$. If we set $\gamma=T_{1}\left(A^{0,1}, \alpha\right)$, then

$$
T_{1}\left(A^{0,1}-2 \bar{\partial} h, \alpha+h \sigma_{0}\right)=\gamma-2 i(\Lambda \bar{\partial} \partial h+\lambda \partial \bar{\partial} h)-2\left|\sigma_{0}\right|^{2} h .
$$

From the Kähler identities, $\Lambda \bar{\partial}=-i \partial^{*}$ and similarly $\Lambda \partial=i \bar{\partial}^{*}$. Thus

$$
i(\Lambda \bar{\partial} \partial h+\lambda \partial \bar{\partial} h)=2 \operatorname{Re} \bar{\partial}^{*} \bar{\partial} h=-\Delta h,
$$

where $\Delta$ is the negative definite Laplacian on $X$, and we seek to solve the equation

$$
2 \Delta h-2\left|\sigma_{0}\right|^{2} h=-\gamma .
$$


(Note that this equation is the linearized version of the Kazdan-Warner equation used in identifying the Seiberg-Witten moduli space with the Hilbert scheme.) Now we have the following:

Lemma 2.3. The operator $\Delta-\left|\sigma_{0}\right|^{2}$ is an isomorphism from $\Omega^{0}(X ; \mathbb{R})$ to itself.

Proof. If $\Delta h-\left|\sigma_{0}\right|^{2} h=0$, then taking the inner product with $h$ we find that $\|d h\|^{2}=\left|\sigma_{0}\right|^{2} h^{2}=0$. Thus $h$ is constant and $\left|\sigma_{0}\right|^{2} h^{2}=0$, so that $h=0$. Hence the operator $\Delta-\left|\sigma_{0}\right|^{2}$ is injective. It is an elliptic operator on $\Omega^{0}(X ; \mathbb{R})$ whose index is the same as the index of the Laplacian on functions, namely zero. Thus it is also surjective.

Thus given the initial representative $\left(A^{0,1}, \alpha\right)$, there is a unique choice of $h$ such that $T_{1}\left(A^{0,1}-2 \bar{\partial} h, \alpha+h \sigma_{0}\right)=0$. Mapping $\left(A^{0,1}, \alpha\right)$ to $\left(A^{0,1}-\right.$ $\left.2 \bar{\partial} h, \alpha+h \sigma_{0}, 0\right)$ then gives a well-defined map from $H^{1}\left(\mathcal{C}_{1}\right)$ to $H^{1}(\mathcal{C})$, and clearly the maps constructed are inverses. We have therefore showed that $H^{1}(\mathcal{C}) \cong H^{1}\left(\mathcal{C}_{1}\right)$. By the proof of Theorem 1.1, there is an exact sequence as claimed in the statement of Theorem 2.1.

We turn now to the identification of $H^{2}(\mathcal{C})$. Given $\gamma \in \Omega^{0}(X ; \mathbb{R})$, it follows from Lemma 2.3 that we can solve the equation $\Delta h-\left|\sigma_{0}\right|^{2} h=$ $\gamma$. Thus there exists an $h$ such that $T_{1}\left(-2 \bar{\partial} h, h \sigma_{0}, 0\right)=\gamma$, and moreover $\left(-2 \bar{\partial} h, h \sigma_{0}, 0\right)$ is in the kernel of $T_{2}+S$. We can therefore identify the cokernel of $\delta_{2}$ with the cokernel of

$$
T_{2}+S: \Omega^{0,1}(X) \oplus \Omega^{0}\left(L_{0}\right) \oplus \Omega^{0,2}\left(L_{0}\right) \rightarrow \Omega^{0,2}(X) \oplus \Omega^{0,1}\left(L_{0}\right) .
$$

Let $\mathcal{K}$ denote the image of $T_{2}+S$, so that $\mathcal{K}$ is the set

$$
\begin{aligned}
& \left\{\left(\bar{\partial} A^{0,1}-\bar{\sigma}_{0} \beta, \bar{\partial} \alpha+\bar{\partial}^{*} \beta+\frac{1}{2} A^{0,1} \sigma_{0}\right):\right. \\
& \left.A^{0,1} \in \Omega^{0,1}(X), \alpha \in \Omega^{0}\left(L_{0}\right), \beta \in \Omega^{0,2}\left(L_{0}\right)\right\} .
\end{aligned}
$$

First consider the subgroup $0 \oplus \operatorname{Ker} \bar{\partial} \subset \Omega^{0,2}(X) \oplus \Omega^{0,1}\left(L_{0}\right)$. For an element in $\mathcal{K} \cap(0 \oplus \operatorname{Ker} \bar{\partial})$ which is the image of $\left(A^{0,1}, \alpha, \beta\right)$, we have $\bar{\partial} A^{0,1}=\bar{\sigma}_{0} \beta$ and $\bar{\partial}\left(\bar{\partial} \alpha+\bar{\partial}^{*} \beta+\frac{1}{2} A^{0,1} \sigma_{0}\right)=0$. By Lemma 2.2 , this condition is equivalent to $\beta=0$ and $\bar{\partial} A^{0,1}=0$. Thus

$$
(0 \oplus \operatorname{Ker} \bar{\partial}) / \mathcal{K} \cap(0 \oplus \operatorname{Ker} \bar{\partial}) \cong \operatorname{Ker} \bar{\partial} /\left\{\bar{\partial} \alpha+\frac{1}{2} A^{0,1} \sigma_{0}: \bar{\partial} A^{0,1}=0\right\} .
$$


This is clearly the same as $H^{1}\left(L_{0}\right) / \sigma_{0} \cdot H^{1}\left(\mathcal{O}_{X}\right)=K_{1}$. So we have found the subspace of $H^{2}(\mathcal{C})$ described in Theorem 2.1. The quotient $K_{2}^{\prime}$ of $H^{2}(\mathcal{C})$ by $K_{1}$ is the same as $\Omega^{0,2}(X) \oplus \operatorname{Im} \bar{\partial} /(\operatorname{Id} \oplus \bar{\partial})(\mathcal{K})$. Now $(\operatorname{Id} \oplus \bar{\partial})(\mathcal{K})$ is the subgroup

$$
\left\{\left(\bar{\partial} A^{0,1}-\bar{\sigma}_{0} \beta, \bar{\partial} \bar{\partial}^{*} \beta+\frac{1}{2} \bar{\partial} A^{0,1} \sigma_{0}\right): A^{0,1} \in \Omega^{0,1}(X), \beta \in \Omega^{0,2}\left(L_{0}\right)\right\} .
$$

If we consider the projection of this subgroup to the factor $\operatorname{Im} \bar{\partial} \subseteq \Omega^{0,2}\left(L_{0}\right)$, it is surjective since $\bar{\partial} \bar{\partial}^{*}$ is an isomorphism on $\operatorname{Im} \bar{\partial}$. Thus $K_{2}^{\prime}$ is isomorphic to

$$
\Omega^{0,2}(X) /\left\{\bar{\partial} A^{0,1}-\bar{\sigma}_{0} \beta: \bar{\partial} \bar{\partial}^{*} \beta+\frac{1}{2} \bar{\partial} A^{0,1} \sigma_{0}=0\right\} .
$$

Let $\mathcal{K}^{\prime}=\left\{\bar{\partial} A^{0,1}-\bar{\sigma}_{0} \beta: \bar{\partial} \bar{\partial}^{*} \beta+\frac{1}{2} \bar{\partial} A^{0,1} \sigma_{0}=0\right\}$. We claim:

Lemma 2.4. $\mathcal{K}^{\prime} \cap \operatorname{Ker} \bar{\partial}^{*}=\left\{-\bar{\sigma}_{0} \beta: \bar{\partial}^{*} \beta=0\right\}$.

Proof. Suppose that

$$
\bar{\partial}^{*} \bar{\partial} A^{0,1}=\bar{\partial}^{*} \bar{\sigma}_{0} \beta ; \quad \bar{\partial} \bar{\partial}^{*} \beta=-\frac{1}{2} \bar{\partial} A^{0,1} \sigma_{0} .
$$

Taking the inner product with $A^{0,1}$, we find:

$$
\begin{aligned}
\left\|\bar{\partial} A^{0,1}\right\|^{2} & =\left\langle\bar{\partial}^{*} \bar{\partial} A^{0,1}, A^{0,1}\right\rangle=\left\langle\bar{\partial}^{*} \bar{\sigma}_{0} \beta, A^{0,1}\right\rangle=\left\langle\bar{\sigma}_{0} \beta, \bar{\partial} A^{0,1}\right\rangle \\
& =\left\langle\beta, \sigma_{0} \bar{\partial} A^{0,1}\right\rangle=-2\left\langle\beta, \bar{\partial} \bar{\partial}^{*} \beta\right\rangle=-2\left\|\bar{\partial}^{*} \beta\right\|^{2} .
\end{aligned}
$$

It follows that $\bar{\partial} A^{0,1}=0$ and that $\bar{\partial}^{*} \beta=0$, and that $\mathcal{K}^{\prime} \cap \operatorname{Ker} \bar{\partial}^{*}$ is as claimed.

Using Lemma 2.4 , there is an injection of $\operatorname{Ker} \bar{\partial}^{*} /\left(\mathcal{K}^{\prime} \cap \operatorname{Ker} \bar{\partial}^{*}\right)$ into $K_{2}^{\prime}$. Now $\operatorname{Ker} \bar{\partial}^{*} \subseteq \Omega^{0,2}(X)$ is naturally $H^{2}\left(\mathcal{O}_{X}\right)$, and $\left\{-\bar{\sigma}_{0} \beta: \bar{\partial}^{*} \beta=\right.$ $0\}=\left\{-\sigma_{0}^{*} \beta: \bar{\partial}^{*} \beta=0\right\}$ is the image of $H^{2}\left(L_{0}\right)$ under $\sigma_{0}^{*}$. The quotient $H^{2}\left(\mathcal{O}_{X}\right) / \sigma_{0}^{*} H^{2}\left(L_{0}\right)$ is isomorphic to the orthogonal complement of $\operatorname{Im} \sigma_{0}^{*}$, namely the kernel of multiplication by $\sigma_{0}$ on $H^{2}\left(\mathcal{O}_{X}\right)$, which we have denoted by $K_{2}$. So there is an injection of $K_{2}$ into $K_{2}^{\prime}$. The real index of $\mathcal{C}$ is $-\operatorname{dim}_{\mathbb{R}} H^{1}(\mathcal{C})+\operatorname{dim}_{\mathbb{R}} H^{2}(\mathcal{C})$, and we have shown that one half the real index is at least

$$
-h^{0}\left(L_{0}\right)+1-\operatorname{dim}_{\mathbb{C}} K_{0}+\operatorname{dim}_{\mathbb{C}} K_{1}+\operatorname{dim}_{\mathbb{C}} K_{2} .
$$


Moreover equality holds only if $K_{2}^{\prime}$ is exactly equal to $\operatorname{Ker} \bar{\partial}^{*} /\left(\mathcal{K}^{\prime} \cap \operatorname{Ker} \bar{\partial}^{*}\right)$, and thus is isomorphic to $K_{2}=\operatorname{Ker}\left\{\times \sigma_{0}: H^{2}\left(\mathcal{O}_{X}\right) \rightarrow H^{2}\left(L_{0}\right)\right\}$. On the other hand, the above alternating sum is the same as $\chi\left(\mathcal{O}_{X}\right)-\chi\left(L_{0}\right)$ (take the alternating sum of the dimensions in the cohomology exact sequence associated to multiplying by $\left.\sigma_{0}\right)$, which as we have seen is one half the real index of $\mathcal{C}$. It follows that $K_{2}^{\prime}=\operatorname{Ker} \bar{\partial}^{*} /\left(\mathcal{K}^{\prime} \cap \operatorname{Ker} \bar{\partial}^{*}\right) \cong K_{2}$ and that we have the desired exact sequence for $H^{2}(\mathcal{C})$.

Corollary 2.5. If in the above notation $X$ is a Kähler surface, $L=K_{X}^{-1}$ with the $\mathrm{Spin}^{c}$ structure corresponding to the trivial line bundle $L_{0}$, then the Zariski tangent space is zero-dimensional and the obstruction space is zero.

Proof. In this case $H^{0}\left(L_{0}\right)=\mathbb{C} \sigma_{0}, \times \sigma_{0}: H^{1}\left(\mathcal{O}_{X}\right) \rightarrow H^{1}\left(L_{0}\right)$ is an isomorphism, and $\sigma_{0} H^{0}\left(K_{X}\right)=H^{0}\left(K_{X}\right)$. Thus both the Zariski tangent space and the obstruction space are zero.

Next we compare the Kuranishi model of the Seiberg-Witten moduli space to the Kuranishi model of the Hilbert scheme described in the previous section. In what follows we assume that $H^{2}\left(\mathcal{O}_{X}\right)=0$. In fact, if $X$ is a minimal surface with $H^{2}\left(\mathcal{O}_{X}\right) \neq 0$, then either $X$ is of general type or it is elliptic, a $K 3$ surface, or a complex torus. In case $X$ is of general type, the relevant Seiberg-Witten moduli spaces are smooth points corresponding to $\pm K_{X}$ of the appropriate dimension, and the Kuranishi obstruction space is zero by Corollary 2.5 above. In case $X$ is elliptic, the moduli space need not be of the expected dimension, and the Kuranishi obstruction space need not be zero, but we shall see in the next section that the obstruction map is always identically zero and hence that the map from $\mathcal{M}_{g}(\xi)$ to $H_{D_{0}, X}$ is a diffeomorphism between two smooth manifolds. The other cases involve reducible solutions to the Seiberg-Witten equations, and thus are slightly exceptional from our point of view. The case of a nonminimal surface may then be reduced to the minimal case, at least for an open set of Kähler metrics; we omit the details. Thus essentially the only interesting case to consider is the case where $H^{2}\left(\mathcal{O}_{X}\right)=0$.

Theorem 2.6. Suppose that $H^{2}\left(\mathcal{O}_{X}\right)=0$. Then the natural homeomorphism from $\mathcal{M}_{g}(\xi)$ to $H_{D_{0}, X}$ is an isomorphism of real analytic spaces.

Proof. We keep the convention that $A$ is a connection on $L=L_{0}^{\otimes 2} \otimes K_{X}$, rather than on $L_{0}$, and that it induces a connection on $L_{0}$ once we have fixed 
once and for all a Hermitian connection on $K_{X}$. Recall that $H_{D_{0}, X}$ is locally defined as the zeroes of the Fredholm map $F(A, \alpha)=\pi_{\text {Ker } \bar{\partial}}\left(\bar{\partial} A+\frac{1}{2} A \cdot \alpha\right)$, restricted to a slice of the complex gauge group action on $\operatorname{Ker} \bar{\partial} \oplus \Omega^{0}\left(L_{0}\right) \subset$ $\Omega^{0,1}\left(L_{0}\right) \oplus \Omega^{0}\left(L_{0}\right)$.

As for $\mathcal{M}_{g}(\xi)$, it is locallly defined by the zero set of the three equations $G=\left(F_{A}^{+}\right)^{1,1}-\frac{i}{2}\left(|\alpha|^{2}-|\beta|^{2}\right) \omega, \bar{\partial} A^{0,1}-\bar{\alpha} \beta$, and $\bar{\partial}_{A} \alpha+\bar{\partial}_{A}^{*} \beta$. Setting the first equation $G$ equal to zero on a slice $S^{\prime}$ for the real gauge group gives a slice for the complex gauge group: indeed, the differential of $G$ is the map $T_{1}$ defined in the proof of Theorem 2.1, and Lemma 2.1 shows that, given $\left(A^{0,1}, \alpha\right)$, there is a unique real-valued $C^{\infty}$ function $h$ such that $T_{1}\left(A^{0,1}-\right.$ $\left.2 \bar{\partial} h, \alpha+h \sigma_{0}\right)=0$. Thus

$$
T_{1}^{-1}(0) \oplus i T \mathcal{G}_{\mathbb{R}}=\Omega^{0,1}(X) \oplus \Omega^{0}\left(L_{0}\right) \oplus \Omega^{0,2}\left(L_{0}\right) .
$$

In particular $S^{\prime} \cap G^{-1}(0)$ is a slice for the complex gauge group in a neighborhood of the origin; denote this slice by $S$.

Consider now the remaining two equations. Defining

$$
\mathcal{F}(A, \alpha, \beta)=\left(\bar{\partial} A^{0,1}-\bar{\alpha} \beta, \bar{\partial}_{A} \alpha+\bar{\partial}_{A}^{*} \beta\right),
$$

we can view $\mathcal{F}$ as a section of the trivial vector bundle over $\Omega^{0,1}(X) \oplus$ $\Omega^{0}\left(L_{0}\right) \oplus \Omega^{0,2}\left(L_{0}\right)$ with fiber $\Omega^{0,2}(X) \oplus \Omega^{0,1}\left(L_{0}\right)$ whose restriction to the slice $S$ is Fredholm and locally defines $\mathcal{M}_{g}(\xi)$.

By our assumption that $H^{2}\left(\mathcal{O}_{X}\right)=0$, and since $H^{2}\left(\mathcal{O}_{X}\right)$ surjects onto $H^{2}\left(L_{0}\right)$, it follows that $\bar{\partial}: \Omega^{0,1}\left(L_{0}\right) \rightarrow \Omega^{0,2}\left(L_{0}\right)$ is surjective. Hence the natural map

$$
\Omega^{0,2}(X) \oplus \Omega^{0,1}\left(L_{0}\right) \rightarrow \operatorname{Ker} \bar{\partial} \oplus \Omega^{0,2}(X) \oplus \Omega^{0,2}\left(L_{0}\right)
$$

defined by $(\psi, \eta) \mapsto\left(\pi_{\text {Ker }} \bar{\partial} \eta \psi, \bar{\partial} \eta\right)$, is an isomorphism. Thus for small $A$, the map

$$
(\psi, \eta) \mapsto\left(\pi_{\mathrm{Ker}} \bar{\partial} \eta, \psi, \bar{\partial}_{A} \eta\right)
$$

is again an isomorphism. We may then view this map as an automorphism of the trivial vector bundle over $\Omega^{0,1}(X) \oplus \Omega^{0}\left(L_{0}\right) \oplus \Omega^{0,2}\left(L_{0}\right)$ (or an appropriate neighborhood of the origin) with fiber $\Omega^{0,2}(X) \oplus \Omega^{0,1}\left(L_{0}\right)$. Under this automorphism, $\mathcal{F}$ corresponds to the section $\left(F_{1}, F_{2}\right)$, where

$$
\begin{aligned}
& F_{1}=\pi_{\text {Ker } \bar{\partial}}\left(\bar{\partial}_{A} \alpha+\bar{\partial}_{A}^{*} \beta\right) ; \\
& F_{2}=\left(\bar{\partial} A^{0,1}-\bar{\alpha} \beta, \bar{\partial}_{A}^{2} \alpha+\bar{\partial}_{A} \bar{\partial}_{A}^{*} \beta\right) .
\end{aligned}
$$

Thus the Kuranishi model for $\mathcal{F}$ on the slice $S$ is the same as that for the pair $\left(F_{1}, F_{2}\right)$ on $S$. It is easy to check that the differential of the map $F_{2}$ is the 
same as the differential of $\mathcal{F}$ followed by $(\mathrm{Id}, \bar{\partial})$. In other words, the cokernel of the differential of $F_{2}$ is exactly the group $K_{2}$ of Theorem 1.1, namely the kernel of multiplication from $H^{2}\left(\mathcal{O}_{X}\right)$ to $H^{2}\left(L_{0}\right)$. Since $H^{2}\left(\mathcal{O}_{X}\right)=0$ by assumption, the differential of $F_{2}$ is onto and $F_{2}^{-1}(0)$ is a smooth submanifold of a neighborhood of the origin in $\Omega^{0,1}(X) \oplus \Omega^{0}\left(L_{0}\right) \oplus \Omega^{0,2}\left(L_{0}\right)$. Now $F_{2}=0$ if and only if $\bar{\partial} A^{0,1}=\bar{\alpha} \beta$ and $\bar{\partial}_{A}^{2} \alpha+\bar{\partial}_{A} \bar{\partial}_{A}^{*} \beta=0$. As we mentioned earlier, these equations imply that $\beta=0$ and hence that $\bar{\partial} A^{0,1}=0$. Conversely, if $\beta=0$ and $\bar{\partial} A^{0,1}=0$, then $F_{2}(A, \alpha, \beta)=0$. Solving the equation $\mathcal{F}=0$ on the slice $S$ is the same then as solving the equation $\pi_{\operatorname{Ker}} \bar{\partial}_{\partial}\left(\bar{\partial}_{A} \alpha\right)=0$ on the slice $S \cap\left(\operatorname{Ker} \bar{\partial} \oplus \Omega^{0}\left(L_{0}\right)\right)$, at least in a neighborhood of the origin. This is exactly the equation $F(A, \alpha)$ on the slice $S \cap\left(\operatorname{Ker} \bar{\partial} \oplus \Omega^{0}\left(L_{0}\right)\right)$. for the complex gauge group. A standard argument (see for example [4], Chapter 4, proof of Theorem 3.8) shows that this is the same as the usual Kuranishi model for $F$, in other words that this model is isomorphic to the Kuranishi model formed by taking any other slice for the $\mathcal{G}_{\mathbb{C}}$-action. Thus the two Kuranishi models are isomorphic as complex spaces.

\section{Obstruction bundles.}

Fix an oriented 4-manifold $X$ with a Riemannian metric $g$ (not necessarily a Kähler surface). Let $\mathcal{A}^{*}(L)$ denote the spaces of pairs $(A, \psi)$, where $A$ is a connection on $L$ and $\psi$ is a nonzero section of $\mathbb{S}^{+}(\xi)$ as in the previous section. The real gauge group $\mathcal{G}$ acts on $\mathcal{A}^{*}(L)$, and we denote the quotient by $\mathcal{B}(L)$. The trivial Hilbert space bundle $i \Omega_{+}^{2}(X ; \mathbb{R}) \oplus \mathbb{S}^{-}(\xi)$ descends to a Hilbert bundle $\mathcal{H}$ over $\mathcal{B}(L)$, and the moduli space $\mathcal{M}_{g}(\xi)$ is the zero set of the Fredholm section $F(A, \psi)$ defined by the Seiberg-Witten equations. As such $\mathcal{M}_{g}(\xi)$ has a real analytic structure and in particular a Zariski tangent space. In this section, we are concerned with the following situation: suppose that the space $\mathcal{M}_{g}(\xi)$ is a smooth compact manifold, not necessarily of the expected dimension. Thus the dimension of the Zariski tangent space of $\mathcal{M}_{g}(\xi)$ at every point is equal to the dimension of $\mathcal{M}_{g}(\xi)$ at that point, and these tangent spaces fit together to form the tangent bundle $T \mathcal{M}_{g}(\xi)$ to $\mathcal{M}_{g}(\xi)$. Note that the tangent bundle is in fact just $\operatorname{Ker} d F: T \mathcal{A}^{*}(L) \rightarrow \mathcal{H}$. It follows that the obstruction spaces have locally constant rank on $\mathcal{M}_{g}(\xi)$ and thus, by standard elliptic theory, fit together to form a vector bundle $\mathcal{O}$ over $\mathcal{M}_{g}(\xi)$. In case $g$ is a Kähler metric on the complex surface $X$, the arguments of the previous section show that the fiber of $\mathcal{O}$ over a point $\left(A_{0}, \sigma_{0}\right)$ may be canonically identified with the middle cohomology of the 
elliptic complex

$$
\Omega^{0,1}(X ; \mathbb{R}) \oplus \Omega^{0}\left(L_{0}\right) \rightarrow \Omega^{0,2}(X ; \mathbb{R}) \oplus \Omega^{0,1}\left(L_{0}\right) \rightarrow \Omega^{0,2}\left(L_{0}\right)
$$

where the first map is $\left(A^{0,1}, \alpha\right) \mapsto\left(\bar{\partial} A^{0,1}, \bar{\partial} \alpha+\frac{1}{2} A^{0,1} \sigma_{0}\right)$ and the second map is $\left.(\varphi, \psi) \mapsto \bar{\partial} \psi-\frac{1}{2} \varphi \cdot \sigma_{0}\right)$. Again by a slight modification of the standard theory for the $\bar{\partial}$-operator, it follows that the bundle $\mathcal{O}$ is a holomorphic vector bundle over $\mathcal{M}_{g}(\xi)$. In fact, letting $\mathfrak{C}$ be the complex $\mathcal{O}_{X \times \mathcal{M}_{g}(\xi)} \rightarrow$ $\mathcal{O}_{X \times \mathcal{M}_{g}(\xi)}\left(\mathcal{D}_{0}\right)$, it is easy to see that $\mathcal{O}$ is the $C^{\infty}$ vector bundle associated to the hyperdirect image $\mathbb{R}^{2} \pi_{2 *} \mathfrak{C} \cong R^{1} \pi_{2 *} \mathcal{O}_{\mathcal{D}_{0}}\left(\mathcal{D}_{0}\right)$. To use this information to evaluate Seiberg-Witten invariants, we have the following:

Theorem 3.1. In the above notation, suppose that the expected real dimension of $\mathcal{M}_{g}(\xi)$ is $2 d$, and let $\mu$ be the natural class in $H^{2}(\mathcal{B}(L))$. Then the value of the Seiberg-Witten function on $\xi$ is $\int_{\mathcal{M}_{g}(\xi)} e(\mathcal{O}) \cup \mu^{d}$, where $e(\mathcal{O})$ is the Euler class of the vector bundle $\mathcal{O}$. In case $X$ is a Kähler surface and $\mathcal{M}_{g}(\xi)$ is equidimensional, this is the same as $\int_{\mathcal{M}_{g}(\xi)} c_{n}(\mathcal{O}) \cup \mu^{d}$, where $n=\operatorname{rank} \mathcal{O}$.

In particular we need to calculate $c_{n}(\mathcal{O})$ :

Lemma 3.2. In the above notation, suppose that $X$ is a Kähler surface and that $\mathcal{M}_{g}(\xi)$ is smooth. Then $c_{n}(\mathcal{O})$ is the degree $n$ term in

$$
c\left(\pi_{2 !} \mathcal{O}_{X \times \mathcal{M}_{g}(\xi)}\left(\mathcal{D}_{0}\right)\right)^{-1} c\left(T \mathcal{M}_{g}(\xi)\right)
$$

Proof. We need to calculate $c_{n}(\mathcal{O})=c_{n}\left(R^{1} \pi_{2 *} \mathcal{O}_{\mathcal{D}_{0}}\left(\mathcal{D}_{0}\right)\right)$. Now the morphism $\mathcal{D}_{0} \rightarrow \mathcal{M}_{g}(\xi)$ has relative dimension one, and so $\pi_{2 !} \mathcal{O}_{\mathcal{D}_{0}}\left(\mathcal{D}_{0}\right)$, which is by definition the alternating sum of the $R^{i} \pi_{2 *} \mathcal{O}_{\mathcal{D}_{0}}\left(\mathcal{D}_{0}\right)$, is just $R^{0} \pi_{2 *} \mathcal{O}_{\mathcal{D}_{0}}\left(\mathcal{D}_{0}\right)-R^{1} \pi_{2 *} \mathcal{O}_{\mathcal{D}_{0}}\left(\mathcal{D}_{0}\right)$. Furthermore, $R^{0} \pi_{2 *} \mathcal{O}_{\mathcal{D}_{0}}\left(\mathcal{D}_{0}\right)$ is just the tangent bundle $T \mathcal{M}_{g}(\xi)$ to $\mathcal{M}_{g}(\xi)$. Thus

$$
c(\mathcal{O})=c\left(R^{1} \pi_{2 *} \mathcal{O}_{\mathcal{D}_{0}}\left(\mathcal{D}_{0}\right)\right)=c\left(\pi_{2 !} \mathcal{O}_{\mathcal{D}_{0}}\left(\mathcal{D}_{0}\right)\right)^{-1} c\left(T \mathcal{M}_{g}(\xi)\right)
$$

Using the exact sequence

$$
0 \rightarrow \mathcal{O}_{X \times \mathcal{M}_{g}(\xi)} \rightarrow \mathcal{O}_{X \times \mathcal{M}_{g}(\xi)}\left(\mathcal{D}_{0}\right) \rightarrow \mathcal{O}_{\mathcal{D}_{0}}\left(\mathcal{D}_{0}\right) \rightarrow 0
$$

it follows that, in the $K$-theory of $\mathcal{M}_{g}(\xi)$,

$$
\pi_{2 !} \mathcal{O}_{\mathcal{D}_{0}}\left(\mathcal{D}_{0}\right)=\pi_{2 !} \mathcal{O}_{X \times \mathcal{M}_{g}(\xi)}\left(\mathcal{D}_{0}\right)-\pi_{2 !} \mathcal{O}_{X \times \mathcal{M}_{g}(\xi)}
$$


Since $\pi_{2 !} \mathcal{O}_{X \times \mathcal{M}_{g}(\xi)}$ is a trivial vector bundle,

$$
c\left(\pi_{2 !} \mathcal{O}_{\mathcal{D}_{0}}\left(\mathcal{D}_{0}\right)\right)=c\left(\pi_{2 !} \mathcal{O}_{X \times \mathcal{M}_{g}(\xi)}\left(\mathcal{D}_{0}\right)\right) .
$$

Putting this together with the above formula for $c(\mathcal{O})$ gives the statement of (3.2).

Proof of (3.1). Consider quite generally the following situation: $\mathcal{H} \rightarrow \mathcal{B}$ is a Hilbert vector bundle over the connected Hilbert manifold $\mathcal{B}$, and $\sigma$ is a smooth section of $\mathcal{H}$. Let $Z=\sigma^{-1}(0)$, assumed connected for the sake of simplicity, and suppose that the differential $d \sigma$ is Fredholm of index $e$, at least in a neighborhood of $Z$. Suppose moreover that $Z$ is a smooth compact submanifold of $\mathcal{B}$ of finite dimension $e^{\prime}$ and that $\operatorname{Ker} d \sigma_{z}$ has constant rank for every $z \in Z$ and that the corresponding subbundle of $T \mathcal{B} \mid Z$ is the tangent bundle to $Z$. Define the obstruction bundle $\mathcal{O} \rightarrow Z$ by $\mathcal{O}=\operatorname{Coker}(d \sigma \mid Z)$, of rank $e^{\prime}-e$. Theorem 3.1 is then a consequence of the following lemma, which implies that the class of a small generic perturbation of $Z$ is the same as the class of a generic section of $\mathcal{O}$, in other words that the Euler class of $\mathcal{O}$ represents the same cohomology class as the Seiberg-Witten class of a generic moduli space.

Lemma 3.3. In the above situation, suppose that $\sigma_{1}$ is a small nonlinear Fredholm $C^{1}$ perturbation of $\sigma$, with $\sigma_{1}^{-1}(0)=Z_{1}$, and that $\sigma_{1}$ is transverse to 0 in the sense that $d \sigma_{1}$ is surjective at every point of $Z_{1}$. Then there exists a section $s$ of $\mathcal{O} \rightarrow Z$ which is transverse to 0 and a small isotopy in $\mathcal{B}$ from $s^{-1}(0)$ to $Z_{1}$.

Proof. Since $Z$ is compact, standard arguments show that there is a neighborhood $\nu$ of $Z$ in $\mathcal{B}$ which is diffeomorphic to a Hilbert disk bundle over $Z$. Let $\pi: \nu \rightarrow Z$ be the retraction. Over $Z$, there is an orthogonal splitting $\mathcal{H} \mid Z \cong \operatorname{Im} d \sigma \oplus \mathcal{O}$. Using $\pi$, we can pull this decomposition back to a splitting of $\mathcal{H} \mid \nu \cong I \oplus \mathcal{O}$. Let $\pi_{1}: \mathcal{H} \mid \nu \rightarrow I$ and $\pi_{2}: \mathcal{H} \mid \nu \rightarrow \mathcal{O}$ be the projections. Consider the composed map $\pi_{1} \circ \sigma: \nu \rightarrow I$. At $z \in Z$, the differential of this map is just $d \sigma$, and so restricted to a fiber $\pi^{-1}(z)$, the differential is an isomorphism. It follows that, if $\nu$ is sufficiently small, then $\pi_{1} \circ \sigma \mid \pi^{-1}(z)$ is an open embedding for all $z \in Z$.

Now let $\sigma_{1}$ be a small perturbation of $\sigma$. If $\sigma_{1}$ is sufficiently close to $\sigma$, then $\sigma_{1}^{-1}(0)=Z_{1} \subseteq \nu$. Consider the map $\pi_{1} \circ \sigma_{1}: \nu \rightarrow I$. If we restrict this map to a fiber $\pi^{-1}(z)$ of the map $\pi: \nu \rightarrow Z, \pi_{1} \circ \sigma_{1} \mid \pi^{-1}(z)$ is close to 
an open embedding. Thus, as long as $\sigma_{1}$ is close to $\sigma, \pi_{1} \circ \sigma_{1} \mid \pi^{-1}(z)$ is also an open embedding. In particular, $\left(\pi_{1} \circ \sigma_{1}\right)^{-1}(0)=\hat{Z}_{1}$ is again a section of $\pi: \nu \rightarrow Z$, and it is close to the zero section $\sigma$. Thus $\hat{Z}_{1}$ is isotopic to $Z$ via a small isotopy in $\nu \subseteq \mathcal{B}$.

Clearly $Z_{1}=\sigma_{1}^{-1}(0)=\left(\pi_{2} \circ \sigma_{1} \mid \hat{Z}_{1}\right)^{-1}(0)$. Moreover $\left(\pi_{2} \circ \sigma_{1} \mid \hat{Z}_{1}\right)$ is a section $\hat{s}$ of the restriction $\mathcal{O} \rightarrow \hat{Z}_{1}$, and, if $\sigma_{1}$ is transverse to 0 , then $\hat{s}$ is also transverse to 0 . Using the isotopy constructed above to identify the section $\hat{s}$ with a section of $\mathcal{O} \rightarrow Z$, we see that we have indeed identified $Z_{1}$ up to isotopy with a transverse section of $\mathcal{O} \rightarrow Z$, as claimed.

There are obvious generalizations of Lemma 3.3, and so of Theorem 3.1. For example, we might only assume that $Z$ is a stratified space with $K$ a compact subset contained in an open subset $U$ of $Z$, such that $U$ is a smooth manifold and $\operatorname{Ker} d \sigma_{u}=T U_{u}$ for all $u \in U$. Then a generic small perturbation $\sigma_{1}$ of $\sigma$ has the property that there exists a neighborhood $N$ of $K$ such that $\sigma_{1}^{-1}(0) \cap N$ is a smooth manifold isotopic to a transverse section of the obstruction bundle over $N$. For example, we might take for $K$ a subset of the form $\mu_{1} \cap \cdots \cap \mu_{k}$, where the $\mu_{i}$ are generic geometric representatives for the $\mu$-divisor. However, we shall not try to formulate the most general possible result along these lines.

\section{Elliptic surfaces.}

Let $X$ denote an elliptic surface. Suppose that $f$ is the divisor class of a general fiber, the multiple fibers are $F_{i}$, and that the multiplicity of $F_{i}$ is $m_{i}$. Thus $K_{X}=\left(p_{g}-1\right) f+\sum_{i}\left(m_{i}-1\right) F_{i}$. We first consider the much simpler case where $X$ is regular, since this case arises in the smooth classification of elliptic surfaces (see for example [5], [2]). Then we will discuss the multiplicities for a general elliptic surface.

If $X$ is regular, $H^{1}\left(\mathcal{O}_{X}\right)=0$ and the Seiberg-Witten obstruction space involves only the two terms $H^{1}\left(\mathcal{O}_{X}\left(D_{0}\right)\right)$ and $H^{2}\left(\mathcal{O}_{X}\left(D_{0}\right)\right)$. The divisor $D_{0}$ is semiregular if and only if $H^{1}\left(\mathcal{O}_{X}\left(D_{0}\right)\right)=0$. It follows from (2.3) of [5] that the $D_{0}$ are exactly the effective divisors which are numerically equivalent to $\frac{1-r}{2} K_{X}$, for a rational number $r \leq 1$. In particular $D_{0} \cdot K_{X}=0$. Another way to describe the $D_{0}$ is that they are the effective divisors numerically equivalent to a rational multiple of $K_{X}$ such that $K_{X}-2 D_{0}$ has positive fiber degree (is a positive rational multiple of the fiber, or equivalently of $K_{X}$ ). A similar statement holds if $X$ is not necessarily assumed to be regular. As $L^{2}=0$, the expected dimension of the moduli space is always zero, i.e. 
$X$ is of simple type. We now compute the dimensions of the cohomology groups:

Lemma 4.1. Suppose that $X$ is regular, and let $D_{0}=a f+\sum_{i} b_{i} F_{i}$ with $a \geq 0$ and $0 \leq b_{i} \leq m_{i}-1$. Then:

$$
\begin{aligned}
& h^{0}\left(D_{0}\right)=a+1 ; \\
& h^{1}\left(D_{0}\right)= \begin{cases}0, & \text { if } a \leq p_{g} \\
a-p_{g}, & \text { if } a \geq p_{g}\end{cases} \\
& h^{2}\left(D_{0}\right)= \begin{cases}p_{g}-a, & \text { if } a \leq p_{g} \\
0, & \text { if } a \geq p_{g}\end{cases}
\end{aligned}
$$

Proof. The statements about $h^{0}\left(D_{0}\right)$ and $h^{2}\left(D_{0}\right)$ are clear and the rest follows from Riemann-Roch, since $\chi\left(\mathcal{O}_{X}\left(D_{0}\right)\right)=1+p_{g}$.

Thus we see that $D_{0}$ is semiregular if and only if $a \leq p_{g}$. However, since $X$ is regular and thus $H_{D_{0}, X}$ is equal to the linear system $\left|D_{0}\right|$, which is a projective space $\mathbb{P}^{a}, H_{D_{0}, X}$ is always smooth and the Zariski tangent space is the actual tangent space. To calculate the value of the Seiberg-Witten invariant on $L$, we take the top Chern class of the obstruction bundle. Now the moduli space is $X \times \mathbb{P}^{a}$, where $\mathbb{P}^{a}=\left|D_{0}\right|$. Over $X \times \mathbb{P}^{a}$, there is the incidence divisor $\mathcal{D}$. The obstruction bundle over $\mathbb{P}^{a}$ has two terms $R^{1} \pi_{2 *} \mathcal{O}_{X \times \mathbb{P}^{a}}(\mathcal{D})$ and $R^{2} \pi_{2 *} \mathcal{O}_{X \times \mathbb{P}^{a}}(\mathcal{D})$.

Proposition 4.2. Suppose in the above notation that $a \leq p_{g}-1$. Then the multiplicity of the Seiberg-Witten invariant is $(-1)^{a}\left(\begin{array}{c}p_{g}-1 \\ a\end{array}\right)$.

Proof. By Lemma 4.1, the first term of the obstruction bundle is zero, and we must compute the top Chern class $c_{a}$ of $\left.R^{2} \pi_{2 *} \mathcal{O}_{X \times \mathbb{P}^{a}}(\mathcal{D})\right)$. Next we calculate the class of $\mathcal{D}$ in $\operatorname{Pic}\left(X \times \mathbb{P}^{a}\right) \cong \pi_{1}^{*} \operatorname{Pic} X \oplus \mathbb{Z} \cdot \pi_{2}^{*} \mathcal{O}_{\mathbb{P}^{a}}(1)$. Since $\mathcal{D}$ is the incidence divisor, its restriction to the slice $\pi_{2}^{*}\{D\}$ is the divisor $D$, whereas its restriction to any slice $\{p\} \times \mathbb{P}^{a}$ such that $p$ is not in the base locus of $D_{0}$ is a hyperplane in $\mathbb{P}^{a}$. Thus $\mathcal{O}_{X \times \mathbb{P}^{a}}(\mathcal{D}) \cong \pi_{1}^{*} \mathcal{O}_{X}\left(D_{0}\right) \otimes \pi_{2}^{*} \mathcal{O}_{\mathbb{P}^{a}}(1)$. So we have

$$
\left.R^{2} \pi_{2 *} \mathcal{O}_{X \times \mathbb{P}^{a}}(\mathcal{D})\right)=R^{2} \pi_{2 *}\left(\pi_{1}^{*} \mathcal{O}_{X}\left(D_{0}\right) \otimes \pi_{2}^{*} \mathcal{O}_{\mathbb{P}^{a}}(1)\right)=\mathcal{O}_{\mathbb{P}^{a}}(1)^{p_{g}-a} .
$$


Setting $h=c_{1}\left(\mathcal{O}_{\mathbb{P}^{a}}(1)\right)$, we want to take the term of degree $a$ in

$$
\left((1+h)^{p_{g}-a}\right)^{-1}=(1+h)^{-\left(p_{g}-a\right)} .
$$

By the binomial theorem (see below for our conventions on binomial coefficients $)$, this is $\left(\begin{array}{c}-\left(p_{g}-a\right) \\ a\end{array}\right)=(-1)^{a}\left(\begin{array}{c}p_{g}-1 \\ a\end{array}\right)$.

A similar argument shows:

Proposition 4.3. Suppose in the above notation that $a \geq p_{g}$. Then the multiplicity of the Seiberg-Witten invariant is 1 if $p_{g}=0$ and is otherwise 0 .

Proof. Since $H^{2}(D)=0$, by Lemma 4.1 , we seek $c_{a}\left(R^{1} \pi_{2 *} \mathcal{O}_{X \times \mathbb{P} a}(\mathcal{D})\right)$. Using the calculation $\mathcal{O}_{X \times \mathbb{P}^{a}}(\mathcal{D}) \cong \pi_{1}^{*} \mathcal{O}_{X}\left(D_{0}\right) \otimes \pi_{2}^{*} \mathcal{O}_{\mathbb{P}^{a}}(1)$ given above, we need to find $c_{a}$ of

$$
R^{1} \pi_{2 *}\left(\pi_{1}^{*} \mathcal{O}_{X}\left(D_{0}\right) \otimes \pi_{2}^{*} \mathcal{O}_{\mathbb{P}^{a}}(1)\right)=H^{1}\left(D_{0}\right) \otimes_{\mathbb{C}} \mathcal{O}_{\mathbb{P}^{a}}(1)=\left(\mathcal{O}_{\mathbb{P}^{a}}(1)\right)^{a-p_{g}} .
$$

If $p_{g}=0$, then $c\left(\left(\mathcal{O}_{\mathbb{P}^{a}}(1)\right)^{a}\right)=(1+h)^{a}$, and thus $c_{a}\left(\left(\mathcal{O}_{\mathbb{P}^{a}}(1)\right)^{a}\right)=1$. Otherwise, the multiplicity is $c_{a}$ of a bundle of rank less than $a$, and so is zero.

Remark. If $p_{g}=0$, then the multiplicity is always 1 , although we can have $a>0$ if there are more than two multiple fibers. If $p_{g}>0$ and there are at most two multiple fibers (the case of finite cyclic fundamental group), then it is easy to check that $a \leq p_{g}-1$. In general however both of the terms in the exact sequence for the obstruction bundle can be nonzero.

Next we turn to elliptic surfaces which are not necessarily regular. To state the result, let us record the following convention on binomial coefficients (made so that the binomial theorem holds): For $m \geq 0$, the binomial coefficient $\left(\begin{array}{c}n \\ m\end{array}\right)=\frac{1}{m !} n(n-1) \cdots(n-m+1)$. Thus it is 1 if $m=0$, by the usual conventions on the empty product, and it is 0 if $0 \leq n<m$ and $m \neq 0$. Moreover $\left(\begin{array}{c}-n \\ m\end{array}\right)=(-1)^{m}\left(\begin{array}{c}n+m-1 \\ m\end{array}\right)$. For example, $\left(\begin{array}{c}-1 \\ m\end{array}\right)=(-1)^{m}$. With this said, suppose that $X$ is a minimal elliptic surface over a smooth curve of genus $g$ and $D_{0}=a f+\sum_{i} b_{i} F_{i}$ is a basic class for $X$. Then the value of the Seiberg-Witten invariant on $D_{0}$ is given by the following formula, due independently to Brussee [2]: 
Proposition 4.4. Let $\pi: X \rightarrow C$ be a minimal elliptic surface over a smooth curve $C$ of genus $g$ and let $D_{0}$ be an effective divisor corresponding to a Seiberg-Witten basic class. Suppose that $D_{0}=\pi^{*} \mathbf{d}+\sum_{i} a_{i} F_{i}$, where $\mathbf{d}$ is an effective divisor on $C$ of degree $d$, the $F_{i}$ are the multiple fibers on $X$, of multiplicity $m_{i}$, and $0 \leq a_{i} \leq m_{i}-1$. Then the multiplicity of the Seiberg-Witten invariant is $(-1)^{d}\left(\begin{array}{c}\bar{\chi}\left(\mathcal{O}_{X}\right)+2 g-2 \\ d\end{array}\right)$.

Proof. If $D_{0}=\pi^{*} \mathbf{d}+\sum_{i} a_{i} F_{i}$ as above, there is a natural morphism from Sym $^{d} C=C_{d}$ to $H_{D_{0}, X}$ obtained by pulling back the universal divisor on $C \times C_{d}$ to $X \times C_{d}$. Slightly tedious arguments left to the reader show that this identifies $H_{D_{0}, X}$ as a set with $C_{d}$. To calculate Zariski tangent spaces, it is easy to see that $H^{0}\left(\mathcal{O}_{D_{0}}\left(D_{0}\right)\right)$ has dimension $d$ by using the fact that the normal bundle of $F_{i}$ is torsion of order exactly $m_{i}$. Thus the dimension of the Zariski tangent space to $H_{D_{0}, X}$ is equal to the dimension of $H_{D_{0}, X}=C_{d}$, and the map $C_{d} \rightarrow H_{D_{0}, X}$ is an isomorphism.

Let $\pi_{2}: X \times C_{d} \rightarrow C_{d}$ be the second projection, let $p: X \times C_{d} \rightarrow C_{d}$ be the map induced by $\pi$, and let $p_{2}: C \times C_{d} \rightarrow C_{d}$ be second projection. Over $C \times C_{d}$ we have the incidence divisor $\mathcal{I}$ defined by $\mathcal{I}=\{(t, \mathbf{d})$ : $t \in \operatorname{Supp} \mathbf{d}\}$. Thus the universal divisor $\mathcal{D}$ on $X \times C_{d}$ is just $p^{*} \mathcal{I}$. Let $\psi_{2}: C \times C_{d} \rightarrow C_{d}$ be projection onto the second factor. By flat base change, $R^{i} \pi_{2 *} p^{*} \mathcal{O}_{C \times C_{d}}(\mathcal{I})=R^{i} \psi_{2 *} \mathcal{O}_{C \times C_{d}}(\mathcal{I})$, and in particular this is zero for $i=2$. Recall that the multiplicity of the Seiberg-Witten invariant is then given by evaluating $c_{a}\left(R^{1} \pi_{2 *} \mathcal{O}_{\mathcal{D}}(\mathcal{D})\right)$ over $C_{d}$. From the exact sequence

$$
0 \rightarrow \mathcal{O}_{X \times C_{d}} \rightarrow \mathcal{O}_{X \times C_{d}}(\mathcal{D}) \rightarrow \mathcal{O}_{\mathcal{D}}(\mathcal{D}) \rightarrow 0,
$$

we see that, in the $K$-theory of $C_{d}$,

$$
\pi_{2 !} \mathcal{O}_{\mathcal{D}}(\mathcal{D})=\sum_{i}(-1)^{i} R^{i} \pi_{2 *} \mathcal{O}_{\mathcal{D}}(\mathcal{D})=R^{0} \pi_{2 *} \mathcal{O}_{\mathcal{D}}(\mathcal{D})-R^{1} \pi_{2 *} \mathcal{O}_{\mathcal{D}}(\mathcal{D})
$$

agrees with $\pi_{2 !} \mathcal{O}_{X \times C_{d}}(\mathcal{D})$ up to the trivial element $\pi_{2 !} \mathcal{O}_{X \times C_{d}}$, and thus they have the same Chern classes. Moreover

$$
c\left(R^{1} \pi_{2 *} \mathcal{O}_{\mathcal{D}}(\mathcal{D})\right)=c\left(\pi_{2 !} \mathcal{O}_{X \times C_{d}}(\mathcal{D})\right)^{-1} c\left(R^{0} \pi_{2 *} \mathcal{O}_{\mathcal{D}}(\mathcal{D})\right) .
$$

Finally $R^{0} \pi_{2 *} \mathcal{O}_{\mathcal{D}}(\mathcal{D})$ is just the tangent bundle $T_{C_{d}}$ to $C_{d}$. By [1], p. 322 ,

$$
c\left(T_{C_{d}}\right)=(1+x)^{d+1-g} e^{-\theta / 1+x},
$$


where $x$ is the class of the divisor $C_{d-1} \subset C_{d}$ and $\theta$ is the pullback of the theta divisor on $\mathrm{Pic}^{d} C$ under the natural map. We also have the formula

$$
\theta^{k} x^{d-k}=\frac{g !}{(g-k) !} .
$$

To find $c\left(\pi_{2 !} \mathcal{O}_{X \times C_{d}}(\mathcal{D})\right)$, we first apply the Grothendieck-Riemann-Roch theorem to find $\operatorname{ch}\left(\pi_{2 !} \mathcal{O}_{X \times C_{d}}(\mathcal{D})\right)$ :

$$
\operatorname{ch}\left(\pi_{2 !} \mathcal{O}_{X \times C_{d}}(\mathcal{D})\right)=\pi_{2 *}\left(\operatorname{ch}\left(\mathcal{O}_{X \times C_{d}}(\mathcal{D})\right) \pi_{1}^{*} \text { Todd } X\right) .
$$

If $\delta=[\mathcal{I}]$ is the class of $\mathcal{I}$ on $C \times C_{d}$, then $\operatorname{ch}\left(\mathcal{O}_{X \times C_{d}}(\mathcal{D})\right)=p^{*} e^{\delta}$. Moreover Todd $X=1+r f+\chi\left(\mathcal{O}_{X}\right) \cdot \mathrm{pt}$ for some rational number $r$. By [1], p. $338, \delta=n[\mathrm{pt}] \otimes 1+\delta^{1,1}+1 \otimes x$, where $x$ is the class defined above and $\left(\delta^{1,1}\right)^{2}=-2[\mathrm{pt}] \otimes \theta,\left(\delta^{1,1}\right)^{2}=\left(\delta^{1,1}\right) \cdot[\mathrm{pt}] \otimes 1=0$. Since $\pi^{*}[\mathrm{pt}]=f$ with $f^{2}=0$, an easy calculation shows that

$$
\operatorname{ch}\left(\pi_{2 !} \mathcal{O}_{X \times C_{d}}(\mathcal{D})\right)=\pi_{2 *}\left(\operatorname{ch}\left(\mathcal{O}_{X \times C_{d}}(\mathcal{D})\right) \pi_{1}^{*} \operatorname{Todd} X\right)=\chi\left(\mathcal{O}_{X}\right) \cdot e^{x}
$$

and thus (setting $\chi\left(\mathcal{O}_{X}\right)=\chi$ for brevity)

$$
c\left(\pi_{2 !} \mathcal{O}_{X \times C_{d}}(\mathcal{D})\right)=(1+x)^{\chi} .
$$

Finally, then, the multiplicity of the Seiberg-Witten invariant is the term of degree $d$ in

$$
(1+x)^{-\chi}(1+x)^{d+1-g} e^{-\theta / 1+x}=(1+x)^{d+1-g-\chi} e^{-\theta / 1+x} .
$$

Let $N=d+1-g-\chi$. Then

$$
\begin{aligned}
(1+x)^{N} e^{-\theta / 1+x} & =\sum_{i}\left(\begin{array}{c}
N \\
i
\end{array}\right) \sum_{j} \frac{1}{j !}(-1)^{j} \theta^{j} \sum_{k}\left(\begin{array}{c}
-j \\
k
\end{array}\right) x^{i+k} \\
& =\sum_{a} \sum_{i+j+k=a}\left(\begin{array}{c}
N \\
i
\end{array}\right) \frac{1}{j !}(-1)^{j}\left(\begin{array}{c}
-j \\
k
\end{array}\right) \frac{g !}{(g-j) !} \\
& =\sum_{a} \sum_{i+j+k=a}(-1)^{j+k}\left(\begin{array}{c}
N \\
i
\end{array}\right)\left(\begin{array}{c}
j+k-1 \\
k
\end{array}\right)\left(\begin{array}{c}
g \\
j
\end{array}\right) .
\end{aligned}
$$

Thus the degree $d$ term is

$$
\sum_{j}(-1)^{j}\left(\sum_{k}(-1)^{k}\left(\begin{array}{c}
N \\
d-j-k
\end{array}\right)\left(\begin{array}{c}
j+k-1 \\
k
\end{array}\right)\right)\left(\begin{array}{l}
g \\
j
\end{array}\right) .
$$

To evaluate the term in parentheses above, we have the straightforward combinatorial lemma: 
Lemma 4.5. We have:

$$
\sum_{k=0}^{a}\left(\begin{array}{c}
a+j+e \\
a-k
\end{array}\right)\left(\begin{array}{c}
-j \\
k
\end{array}\right)=\sum_{k=0}^{a}(-1)^{k}\left(\begin{array}{c}
a+j+e \\
a-k
\end{array}\right)\left(\begin{array}{c}
j+k-1 \\
k
\end{array}\right)=\left(\begin{array}{c}
a+e \\
a
\end{array}\right) .
$$

(By our conventions on binomial coefficients, this is 1 if $a=0$ and is zero for $-a \leq e<0$ and $a \neq 0$.)

Proof. This follows by comparing the coefficient of $t^{a}$ in the two different power series expansions of $(1+t)^{a+e}=(1+t)^{a+e+j}(1+t)^{-j}$.

Returning to the proof of (4.4), the lemma shows that the term in parentheses is $\left(\begin{array}{c}N-j \\ N-j-e\end{array}\right)$, where $e=1-g-\chi($ take $a=d-j$ and $N=a+j+e)$. Thus we obtain

$$
\begin{aligned}
\sum_{j}(-1)^{j}\left(\begin{array}{c}
N-j \\
N-j-e
\end{array}\right)\left(\begin{array}{c}
g \\
j
\end{array}\right) & =\sum_{j}(-1)^{j}\left(\begin{array}{c}
d-j+1-g-\chi \\
d-j
\end{array}\right)\left(\begin{array}{l}
g \\
j
\end{array}\right) \\
& =(-1)^{d} \sum_{j}\left(\begin{array}{c}
\chi+g-2 \\
d-j
\end{array}\right)\left(\begin{array}{c}
g \\
j
\end{array}\right),
\end{aligned}
$$

which is just $(-1)^{d}$ times the coefficient of $t^{d}$ in $(1+t)^{\chi+g-2}(1+t)^{g}=$ $(1+t)^{\chi+2 g-2}$, namely $(-1)^{d}\left(\begin{array}{c}\chi+2 g-2 \\ d\end{array}\right)$, as claimed.

\section{Product ruled surfaces.}

In this section we shall consider the ruled surfaces $X$ of the form $\mathbb{P}^{1} \times C$, where $C$ is a curve of genus $g \geq 1$. We shall also always assume that $C$ is a generic curve in the sense of Brill-Noether theory, and shall use [1] as a general reference for the theory of special divisors on curves.

Let $\pi_{1}: X=\mathbb{P}^{1} \times C \rightarrow \mathbb{P}^{1}$ be the projection onto the first factor and let $\pi_{2}: \mathbb{P}^{1} \times C \rightarrow C$ be the projection onto the second. Let $F_{1}=\pi_{2}^{-1}\{p\}$ be a fiber isomorphic to $\mathbb{P}^{1}$ and let $F_{2}=\pi_{1}^{-1}\{p\}$ be a fiber isomorphic to $C$. Thus $F_{i}^{2}=0$ and $F_{1} \cdot F_{2}=1$. In general we shall refer to a divisor numerically equivalent to $n F_{1}+m F_{2}$ as a divisor of type $(n, m)$, and similarly for a complex line bundle. Thus for example $K_{X}$ is of type $(2 g-2,-2)$. Hence $K_{X}^{2}=-8(g-1)$. Let $L$ be a line bundle of type $(2 a, 2 b)$, so that 
$c_{1}(L) \equiv K_{X} \bmod 2$. Then $L^{2}=8 a b$, and so $L^{2} \geq K_{X}^{2}$ if and only if $a b \geq 1-g$. Next suppose that $L_{0}=\left(K_{X} \otimes L\right)^{1 / 2}$ has a holomorphic section for some holomorphic structure on $L_{0}$. As $L_{0}$ is of type $(g-1+a, b-1)$, we must have $b \geq 1$ and $a \geq 1-g$, and we can write $L_{0}=\mathcal{O}_{X}\left(D_{0}\right)$, where $D_{0}$ is linearly equivalent to $(b-1) \pi_{1}^{*}(\mathrm{pt})+\pi_{2}^{*} \mathbf{d}$ for some divisor $\mathbf{d}$ on $C$ of degree $d=g-1+a$.

Next we turn to the condition that $L \cdot \omega<0$ for some Kähler form $\omega$. The real cohomology classes of Kähler metrics are exactly the classes $\omega$ of type $(x, y)$ with $x, y \in \mathbb{R}$, and $x, y>0$. Thus

$$
\omega \cdot L=2 x b+2 a y .
$$

Since $b \geq 1$, we must have $a<0$, and it is clear that by choosing $x / y<-a / b$, we can then arrange $\omega \cdot L<0$. (Note conversely that if $x / y>-a / b$, then $L$ does not correspond to a basic class. Since $-a \leq g-1$ and $b \geq 1$, if we choose $x / y \geq g-1$, then there are no basic classes.) The final conditions on $a$ and $b$ are:

$$
b \geq 1 ; \quad \frac{1-g}{b} \leq a<0 .
$$

We note that the expected (complex) dimension of the Seiberg-Witten moduli space is $g-1+a b$. However, as we shall see, the actual dimension is equal to the expected dimension only for $b=1$.

Given a curve $D_{0}$ of type $(g-1+a, b-1)=(d, b-1)$, its irreducible components correspond to curves of type $\left(e_{i}, c_{i}\right)$ with $\sum_{i} e_{i}=d$ and $\sum_{i} c_{i}=$ $b-1$. For example, if $D_{0}$ is irreducible, then $D_{0}$ is simultaneously a cover of $\mathbb{P}^{1}$ of degree $d$ and a cover of $C$ of degree $b-1$. If $b=1$, then necessarily $D_{0}$ is a union of $d$ copies of $C$, or more precisely a divisor of the form $\pi_{2}^{*} \mathrm{~d}$ for some divisor $\mathbf{d}$ of degree $d$ on $C$. In this case, $H_{D_{0}, X}$ is just $C_{d}$, the $d^{\text {th }}$ symmetric product of $C$ with itself. Note that $-K_{X}$ is a divisor of this type, with $a=1-g$. In general, for such divisors, we have:

Proposition 5.1. In case $c_{1}(L)=2 a F_{1}+2 F_{2}$, then $H_{D_{0}, X}=C_{d}$ and the value of the Seiberg-Witten invariant is 1.

Proof. We have seen that $H_{D_{0}, X}=C_{d}$ as sets. There is an obvious universal divisor on $X \times C_{d}$ which is the pullback of the universal divisor on $C \times C_{d}$. Thus there is a morphism from $C_{d}$ to $H_{D_{0}, X}$. To see that this morphism is an isomorphism of schemes, it will suffice to show that $H_{D_{0}, X}$ is smooth of dimension $d$. It is an easy exercise to identify $H^{i}\left(\mathcal{O}_{D_{0}}\left(D_{0}\right)\right)$ with $H^{i}\left(\mathbb{P}^{1} ; H^{0}\left(\mathcal{O}_{\mathbf{d}}\right) \otimes \mathcal{O}_{\mathbb{P}^{1}}\right)=H^{i}\left(\mathbb{P}^{1} ; \mathcal{O}_{\mathbb{P}^{1}}\right) \otimes H^{0}\left(\mathcal{O}_{\mathbf{d}}\right)$. This has dimension 
$d$ for $i=0$ and is zero for $i=1$. Thus $H_{D_{0}, X}$ is smooth of dimension $d$, and is therefore isomorphic to $C_{d}$. Note that $X$ is not of simple type if $d>0$.

Clearly, for $p \in X$ with $\pi_{2}(p)=t \in C$, the incidence divisor for $H_{D_{0}, X}$ and $p \in X$ may be identified with the incidence divisor for $C_{d}$ and $t \in C$, along with multiplicities. Let $x$ be the class of the divisor in $C_{d}$. By choosing $d$ distinct points $t_{1}, \ldots, t_{d}$ and checking that the intersections are transverse, we see that $x^{d}=1$. Thus $\mu^{d}=1$ for the Seiberg-Witten moduli space as well, so that the value of the invariant is 1 .

For the remainder of this section, we shall mainly be interested in the case $b=2$. In this case every curve $D_{0}$ of type $(d, 1)$ can be written either as $D_{0}=D_{1}+\pi_{2}^{*} \mathbf{d}_{2}$, where $D_{1}$ is the graph of a map $C \rightarrow \mathbb{P}^{1}$ of degree $d_{1}$ and $\mathrm{d}_{2}$ is a divisor of degree $d_{2}=d-d_{1}$ on $C$, or $D_{0}=\pi_{1}^{*}(\mathrm{pt})+\pi_{2}^{*} \mathrm{~d}$, where $\mathrm{d}$ is a divisor of degree $d$ on $C$. More generally, an irreducible divisor $D_{0} \subset \mathbb{P}^{1} \times C$ of type $(d, b-1)$ which is a section of the line bundle $\pi_{1}^{*} \mathcal{O}_{\mathbb{P}^{1}}(b-1) \otimes \pi_{2}^{*} \mathcal{O}_{C}(\mathbf{d})$ corresponds to a map $C \rightarrow \operatorname{Sym}^{b-1} \mathbb{P}^{1} \cong \mathbb{P}^{b-1}$. In this case it is easy to check that the pullback of $\mathcal{O}_{\mathbb{P}^{b-1}}(1)$ to $C$ is just $\mathcal{O}_{C}(\mathbf{d})$. Moreover, let $V$ be the smallest linear subspace of $\mathbb{C}^{b}$ such that $\mathbb{P}(V) \subseteq \mathbb{P}^{b-1}$ contains the image of $C$. Then $V$ is naturally a quotient of $H^{0}(\mathbf{d})^{*}$, of dimension $r+1$, say, corresponding to a linear subseries of $|\mathbf{d}|$. Note in particular that we always have $r \leq b-1$.

Next suppose that $D_{0}$ is not necessarily irreducible. Then $D_{0}$ still corresponds to a linear system $V \subseteq H^{0}(\mathbf{d})^{*}$ with $\mathbb{P}(V) \subseteq \operatorname{Sym}^{b-1} \mathbb{P}^{1} \cong \mathbb{P}^{b-1}$. In fact, if $D_{0}$ is defined by the section $\sigma_{0} \in H^{0}(\mathbf{b}) \otimes H^{0}(\mathbf{d})$, write $\sigma_{0}=\sum_{i} \alpha_{i} \otimes \beta_{i}$, where the $\alpha_{i} \in H^{0}(\mathbf{b})$ and the $\beta_{i} \in H^{0}(\mathbf{d})$ are linearly independent. For $p \in C$, the morphism $C \rightarrow \operatorname{Sym}^{b-1} \mathbb{P}^{1}$ sends $p$ to $\sum_{i} \beta_{i}(p) \alpha_{i}$, after choosing a coordinate for $\mathcal{O}_{C}(\mathbf{d})$ at $p$. This is well-defined if $p$ is not in the base locus of the span of the $\beta_{i}$, and extends to a unique morphism $C \rightarrow \mathrm{Sym}^{b-1} \mathbb{P}^{1}$. Now $\sigma_{0} \in H^{0}(\mathbf{b}) \otimes H^{0}(\mathbf{d})$ defines a homomorphism $H^{0}(\mathbf{d})^{*} \rightarrow H^{0}(\mathbf{b})$ whose image $V$ is spanned by the $\alpha_{i}$. Thus $V^{*} \subseteq H^{0}(\mathbf{d})$ is a linear series.

At one extreme, consider divisors $D_{0}$ of the form $\pi_{1}^{*} \mathbf{b}+\pi_{2}^{*} \mathbf{d}$, where $\operatorname{deg} \mathbf{b}=b-1$ and $\operatorname{deg} \mathbf{d}=d$. In this case, $V$ has dimension 1 , the linear series corresponding to $V$ is the single divisor $\mathbf{d}$, which is the base locus for the series, and the map $C \rightarrow \operatorname{Sym}^{b-1} \mathbb{P}^{1} \cong \mathbb{P}^{b-1}$ is constant, with image equal to b. In this case, let $\mathfrak{M}_{0}=\mathbb{P}^{b-1} \times C_{d}$, thinking of this space as parametrizing all divisors of the form $\pi_{1}^{*} \mathrm{~b}+\pi_{2}^{*} \mathrm{~d}$. Then we have an obvious divisor on the product $X \times \mathfrak{M}_{0}$, and thus there is an injective morphism $\mathbb{P}^{b-1} \times C_{d} \rightarrow H_{D_{0}, X}$. In paiticular, $H_{D_{0}, X}$ has dimension at least $d+b-1=g-1+a+b-1=$ $g+a+b-2$, whereas the expected dimension of $H_{D_{0}, X}$ is $g+a b-1$. In this 
case the difference between $\operatorname{dim} \mathfrak{M}_{0}$ and the expected dimension of $H_{D_{0}, X}$ is $(b-1)(1-a)$. Since $a<0$ we see that the actual dimension is always greater than the expected dimension as long as $b>1$. To see the image of the tangent space to $\mathfrak{M}_{0}$ inside the Zariski tangent space of $H_{D_{0}, X}$, note that the Zariski tangent space of $H_{D_{0}, X}$ is $H^{0}\left(\mathcal{O}_{D_{0}}\left(D_{0}\right)\right)$. In case $D_{0}=\pi_{1}^{*} \mathbf{b}+\pi_{2}^{*} \mathbf{d}$, there is an map $H^{0}\left(\mathcal{O}_{\pi_{1}^{*} \mathbf{b}}\right) \oplus H^{0}\left(\mathcal{O}_{\pi_{2}^{*} \mathrm{~d}}\right) \rightarrow H^{0}\left(\mathcal{O}_{D_{0}}\left(D_{0}\right)\right)$ given as follows: If we set $E_{1}=\pi_{1}^{*} \mathrm{~b}$ and $E_{2}=\pi_{2}^{*} \mathrm{~d}$, then this is just the natural map

$$
\left(\mathcal{O}_{X}\left(E_{1}\right) / \mathcal{O}_{X}\right) \oplus\left(\mathcal{O}_{X}\left(E_{2}\right) / \mathcal{O}_{X}\right) \rightarrow \mathcal{O}_{X}\left(E_{1}+E_{2}\right) / \mathcal{O}_{X} .
$$

Locally for $R=\mathbb{C}\left\{z_{1}, z_{2}\right\}$, this is the same as the map

$$
R / z_{1}^{a_{1}} R \oplus R / z_{2}^{a_{2}} R \rightarrow R / z_{1}^{a_{1}} z_{2}^{a_{2}} R
$$

defined by $(f, g) \mapsto z_{2}^{a_{2}} f+z_{1}^{a_{1}} g$, which is an inclusion since $z_{1}^{a_{1}}$ and $z_{2}^{a_{2}}$ are relatively prime. Thus the image of the tangent space of $\mathfrak{M}_{0}$ is $L_{0}=$ $H^{0}\left(\mathcal{O}_{\pi_{1}^{*} \mathbf{b}}\right) \oplus H^{0}\left(\mathcal{O}_{\pi_{2}^{*} \mathbf{d}}\right)$, of dimension $b-1+d$, and the map from $\mathfrak{M}_{0}$ to $H_{D_{0}, X}$ is an immersion.

Concerning the structure of $H_{D_{0}, X}$ at a divisor $D_{0}$ of the form $\pi_{1}^{*} \mathbf{b}+\pi_{2}^{*} \mathbf{d}$, where $\operatorname{deg} \mathbf{b}=b-1$ and $\operatorname{deg} \mathbf{d}=d=g-1+a$, we have the following result:

Lemma 5.2. The scheme $H_{D_{0}, X}$ is smooth of dimension $(b-1)(1-a)$ at the divisor $D_{0}=\pi_{1}^{*} \mathbf{b}+\pi_{2}^{*} \mathbf{d}$ if and only if $h^{0}(C ; \mathbf{d})=1$, or in other words if and only if $\mathbf{d}$ is an effective divisor which does not move in a nontrivial linear system. More generally, if $\operatorname{dim}|\mathbf{d}|=r$ and if $T$ is the Zariski tangent space of $H_{D_{0}, X}$ at $D_{0}$, then there is an exact sequence

$0 \rightarrow\left(H^{0}\left(\mathbb{P}^{1} ; \mathbf{b}\right) \otimes H^{0}(C ; \mathbf{d})\right) / \mathbb{C} \sigma_{0} \rightarrow T \rightarrow \operatorname{Ker}\left\{H^{1}\left(\mathcal{O}_{C}\right) \rightarrow H^{1}\left(\mathcal{O}_{C}(\mathbf{d})\right)\right\} \rightarrow 0$, where the map $H^{1}\left(\mathcal{O}_{C}\right) \rightarrow H^{1}\left(\mathcal{O}_{C}(\mathbf{d})\right)$ is given by multiplying by the section coresponding to $\mathbf{d}$. Thus

$$
\operatorname{dim} T=b(r+1)-1+d-r .
$$

Finally, the image of $\mathfrak{M}_{0}$ is a component of $H_{D_{0}, X}$.

Proof. First note that $H^{0}\left(X ; \mathcal{O}_{X}\left(D_{0}\right)\right)=H^{0}\left(\mathbb{P}^{1} ; \mathcal{O}_{\mathbb{P}^{1}}(b-1)\right) \otimes H^{0}(C ; \mathbf{d})$. Thus if $h^{0}(C ; \mathbf{d})=1$, then every nonzero section of $H^{0}\left(X ; \mathcal{O}_{X}\left(D_{0}\right)\right)$ is of the form $\pi_{1}^{*} \mathbf{b}+\pi_{2}^{*} \mathbf{d}$ for some divisor $\mathbf{b}$ of degree $b-1$ on $\mathbb{P}^{1}$. Hence $h^{0}\left(D_{0}\right)=b$ and $\left|D_{0}\right| \cong \mathbb{P}^{b-1}$. In general, setting $r+1=h^{0}(\mathbf{d})$,

$$
\operatorname{dim} H^{0}\left(\mathcal{O}_{X}\left(D_{0}\right)\right) / \mathbb{C} \cdot \sigma_{0}=b(r+1)-1 .
$$


Now $H^{1}\left(\mathcal{O}_{X}\right) \cong H^{0}\left(\mathcal{O}_{\mathbb{P}^{1}}\right) \otimes H^{1}\left(\mathcal{O}_{C}\right)$. Given a section $\sigma_{0}$ of $\mathcal{O}_{X}\left(D_{0}\right)$ of the form $\sigma_{1} \otimes \sigma_{2}$, multiplication by $\sigma_{0}$ is just multiplication by $\sigma_{2}$ from $H^{1}\left(\mathcal{O}_{C}\right)$ to $H^{1}(\mathbf{d})$, followed by multiplication by $\sigma_{1}$. On the other hand, using the exact sequence

$$
0 \rightarrow \mathcal{O}_{C} \rightarrow \mathcal{O}_{C}(\mathbf{d}) \rightarrow \mathcal{O}_{\mathbf{d}} \rightarrow 0
$$

where the map $\mathcal{O}_{C} \rightarrow \mathcal{O}_{C}(\mathbf{d})$ is multiplication by $\sigma_{2}$, it follows that the dimension of the kernel of the map $H^{1}\left(\mathcal{O}_{C}\right) \rightarrow H^{1}(\mathbf{d})$ is $d-r$ (and the dimension of the cokernel is $g-d+r$ ). So the dimension of the Zariski tangent space is $b(r+1)-1+d-r$, whereas the actual dimension of $\mathfrak{M}_{0}$ is $b-1+d$. For the generic divisor $\mathbf{d} \in C_{d}, r=0$, since $d=g+a-1<g$. For such a divisor $\mathbf{d}$, the map $\mathfrak{M}_{0} \rightarrow H_{D_{0}, X}$ is an embedding near $\mathbf{d}$. Thus the image of $\mathfrak{M}_{0}$ is a component of $H_{D_{0}, X}$. Finally $H_{D_{0}, X}$ is smooth at $\mathbf{d} \in \mathfrak{M}_{0}$ if and only if $r=0$.

The same argument identifies the obstruction space at $D_{0}$ :

Lemma 5.3. Suppose as above that $D_{0}=\pi_{1}^{*} \mathbf{b}+\pi_{2}^{*} \mathbf{d}$. Then

$$
\text { Coker }\left\{\times \sigma_{0}: H^{1}\left(\mathcal{O}_{X}\right) \rightarrow H^{1}\left(\mathcal{O}_{X}\left(D_{0}\right)\right)\right\}
$$

is equal to $H^{0}\left(\mathcal{O}_{\mathbb{P}^{1}}(b-1)\right) / \mathbb{C} \cdot \sigma_{1} \otimes H^{1}(\mathbf{d})$ and has dimension $(b-1)(g-d+r)$.

We can put Lemma 5.3 in a more intrinsic global form as follows. The tangent bundle to $\mathbb{P}^{b-1}$ is naturally $\mathcal{O}_{\mathbb{P}^{b-1}}(1)^{b} / \mathcal{O}_{\mathbb{P}^{b-1}}$. Over $C \times C_{d}$ we have the incidence divisor $\mathcal{I}$ defined by

$$
\mathcal{I}=\{(t, \mathbf{d}): t \in \operatorname{Supp} \mathbf{d}\} .
$$

Let $\psi_{2}: C \times C_{d} \rightarrow C_{d}$ be projection onto the second factor. Then using the exact sequence

$$
0 \rightarrow \mathcal{O}_{C \times C_{d}} \rightarrow \mathcal{O}_{C \times C_{d}}(\mathcal{I}) \rightarrow \mathcal{O}_{\mathcal{I}}(\mathcal{I}) \rightarrow 0
$$

we have an exact sequence of direct image sheaves by applying $R^{i} \psi_{2 *}$. Here $\psi_{2} \mid \mathcal{I}$ is a $d$-sheeted cover, and in particular it is finite. Moreover $\mathcal{I}$ is a hypersurface in $C \times C_{d}$ and $\mathcal{I} \cap \psi_{2}^{-1}(\mathbf{d})$ is identified with the divisor $\mathbf{d}$ on $C$. It is easy to see that $R^{0} \psi_{2 *} \mathcal{O}_{\mathcal{I}}(\mathcal{I})$ is canonically the tangent bundle $T_{C_{d}}$ of $C_{d}$. Since it is torsion free, and $R^{0} \psi_{2 *} \mathcal{O}_{C \times C_{d}} \rightarrow R^{0} \psi_{2 *} \mathcal{O}_{C \times C_{d}}(\mathcal{I})$ is an isomorphism at a general $\mathbf{d}, R^{0} \psi_{2 *} \mathcal{O}_{C \times C_{d}} \cong R^{0} \psi_{2 *} \mathcal{O}_{C \times C_{d}}(\mathcal{I})$ and we have an exact sequence

$$
0 \rightarrow T_{C_{d}} \rightarrow R^{1} \psi_{2 *} \mathcal{O}_{C \times C_{d}} \rightarrow R^{1} \psi_{2 *} \mathcal{O}_{C \times C_{d}}(\mathcal{I}) \rightarrow 0 .
$$


Here $R^{1} \psi_{2 *} \mathcal{O}_{C \times C_{d}}=H^{1}\left(\mathcal{O}_{C}\right) \times \mathcal{O}_{C_{d}}$ is a trivial bundle of rank $g$ on $C_{d}$, and we set $\mathcal{E}=R^{1} \psi_{2 *} \mathcal{O}_{C \times C_{d}}(\mathcal{I})$. Note that the Chern classes of $\mathcal{E}$ are given by $c(\mathcal{E})=c\left(T_{C_{d}}\right)^{-1}$.

Lemma 5.4. Let $p_{1}: \mathbb{P}^{b-1} \times C_{d} \rightarrow \mathbb{P}^{b-1}$ be projection onto the first factor and let $p_{2}: \mathbb{P}^{b-1} \times C_{d} \rightarrow C_{d}$ be projection onto the second factor. We then have a map $p_{1}^{*} T_{\mathbb{P}^{b-1}} \otimes p_{2}^{*} \mathcal{E} \rightarrow R^{1} \pi_{2 *} \mathcal{O}_{X \times \mathfrak{M}_{0}}(\mathcal{D}) / R^{1} \pi_{2 *} \mathcal{O}_{X \times \mathfrak{M}_{0}}$, and it is an isomorphism over those points $(\mathbf{b}, \mathbf{d})$ of $\mathfrak{M}_{0}$ where $h^{0}(\mathbf{d})=1$.

Let us study the obstruction space for a divisor $D_{0}$ which is not necessarily in $\mathfrak{M}_{0}$.

Lemma 5.5. Let $D_{0} \subset \mathbb{P}^{1} \times C$ be a divisor of type $(d, b-1)$, corresponding to a morphism $C \rightarrow \operatorname{Sym}^{b-1} \mathbb{P}^{1} \cong \mathbb{P}^{b-1}$. Let $V \subset H^{0}(\mathbf{d})^{*}$ be the linear subspace corresponding to the image of $C$. Then the obstruction space for $H_{D_{0}, X}$ at $D_{0}$ is zero if and only if the map $C \rightarrow \mathbb{P}^{b-1}$ is nondegenerate and the map

$$
\mu_{0}: V^{*} \otimes H^{0}\left(K_{C}-\mathbf{d}\right) \rightarrow H^{0}\left(K_{C}\right)
$$

given by cup product is injective, which holds for a generic curve $C$.

Proof. The obstruction space is given as the cokernel of the map $H^{1}\left(\mathcal{O}_{X}\right) \rightarrow$ $H^{1}\left(\mathcal{O}_{X}\left(D_{0}\right)\right)$ which is multiplication by $\sigma_{0}$. Here $H^{1}\left(\mathcal{O}_{X}\right) \cong H^{0}\left(\mathcal{O}_{\mathbb{P}^{1}}\right) \otimes$ $H^{1}\left(\mathcal{O}_{C}\right)$ and $H^{1}\left(\mathcal{O}_{X}\left(D_{0}\right)\right) \cong H^{0}\left(\mathcal{O}_{\mathbb{P}^{1}}(b-1)\right) \otimes H^{1}\left(\mathcal{O}_{C}(\mathbf{d})\right)$. Let $V$ be the quotient of $H^{0}(\mathbf{d})^{*}$ corresponding to the map $\sigma_{0} \in H^{0}(\mathbf{b}) \otimes H^{0}(\mathbf{d})=$ $\operatorname{Hom}\left(H^{0}(\mathbf{d})^{*}, H^{0}(\mathbf{b})\right)$, and let $\alpha_{1}, \ldots, \alpha_{n}$ be a basis for $V$ viewed as a subspace of $H^{0}(\mathbf{b})$. It follows that we can write $\sigma_{0}=\sum_{i} \alpha_{i} \otimes \beta_{i}$ for $\beta_{i} \in H^{0}(\mathbf{d})$ which are linearly independent. In this case, $\left\{\beta_{i}\right\}$ must also be a basis for $V^{*} \subseteq H^{0}(\mathbf{d})$. Multiplication by $\sigma_{0}$ is equivalent to the map sending $\xi \in H^{1}\left(\mathcal{O}_{C}\right)$ to $\sum_{i} \alpha_{i} \otimes\left(\beta_{i} \xi\right)$. This map is surjective if and only if $V=H^{0}\left(\mathcal{O}_{\mathbb{P}^{1}}(b-1)\right)$ and the natural map

$$
H^{1}\left(\mathcal{O}_{C}\right) \rightarrow H^{0}\left(\mathcal{O}_{\mathbb{P}^{1}}(b-1)\right) \otimes H^{1}(\mathbf{d})
$$

is surjective. After composing with an invertible linear map from $H^{0}\left(\mathcal{O}_{\mathbb{P}^{1}}(b-\right.$ 1)) to itself, we can assume that $\alpha_{i}=\beta_{i}^{*}$, the dual basis to $\beta_{i}$. In this case multiplication by $\sigma_{0}$ is easily seen to be the adjoint of the map $\mu_{0}$. Thus multiplication by $\sigma_{0}$ is surjective if and only if $V=H^{0}\left(\mathcal{O}_{\mathbb{P}^{1}}(b-1)\right)$ and $\mu_{0}$ is injective. 
We note that, in case $\mu_{0}$ is injective, and in particular for a generic curve $C,(5.5)$ identifies the obstruction space as $\left(H^{0}\left(\mathcal{O}_{\mathbb{P}^{1}}(b-1)\right) / V\right) \otimes H^{1}(\mathbf{d})$. For example, in case $\sigma_{0}=\pi_{1}^{*} \mathbf{b}+\pi_{2}^{*} \mathrm{~d}, V$ is a line in $H^{0}\left(\mathcal{O}_{\mathbb{P}^{1}}(b-1)\right)$ and the obstruction space has dimension $(b-1)(g-d+r)$ as given by Lemma 5.3. In case $b=2$ and $\mu_{0}$ is injective, the only possibilities are $\operatorname{dim} V=$ 1 corresponding to $\sigma_{0}=\pi_{1}^{*} \mathbf{b}+\pi_{2}^{*} \mathbf{d}$, and $\operatorname{dim} V=2, V=H^{0}\left(\mathcal{O}_{\mathbb{P}^{1}}(1)\right)$, corresponding to $D_{0}=D_{1}+\pi_{2}^{*} \mathrm{~d}^{\prime}$, where $D_{1}$ is the graph of a nonconstant map from $C$ to $\mathbb{P}^{1}$. Thus the obstruction space is necessarily zero in this case, provided that $\mu_{0}$ is injective.

Let us now describe all of the components of $H_{D_{0}, X}$ in the case $b=2$. In this case $0<-a<(g-1) / 2$ and so $d>(g-1) / 2$. Now suppose that $D_{0}=D_{1}+\pi_{2}^{*} \mathrm{~d}^{\prime}$, where $D_{1}$ is the graph of a map from $C$ to $\mathbb{P}^{1}$ of degree $d_{1}$ and $\mathbf{d}^{\prime}$ has degree $d^{\prime}=d-d_{1}$. Thus $D_{1}$ corresponds to a linear subseries of $\left|\mathbf{d}^{\prime}\right|$, which we can write as $\mathbb{P}\left(V^{*}\right)$ for some vector space $V$ of dimension two, together with a choice of isomorphism $V \cong \mathbb{P}^{1}$. In general we can think of $D_{0}$ as corresponding to a sublinear system of $|\mathrm{d}|$ with base points. If $C$ is generic in the Brill-Noether sense, then for there to exist a map from $C$ to $\mathbb{P}^{1}$ of degree $d_{1}$, we must have the Brill-Noether number $\rho=2 d_{1}-g-2 \geq 0$, in which case the set $G_{d}^{1}$ of all linear series of degree $d$ and dimension one has dimension exactly $\rho$. Moreover for a generic curve $C$, if $\rho=0$ then $G_{d}^{1}$ consists of reduced points, whereas if $\rho>0$ then $G_{d}^{1}$ is smooth and irreducible of dimension of dimension $\rho$ and the generic linear series in $G_{d}^{1}$ is complete. We see then that if $d=(g-1) / 2, g / 2,(g+1) / 2$, then $\mathfrak{M}_{0}$ is the unique component of $H_{D_{0}, X}$. For $d=(g+2) / 2$ the components of $H_{D_{0}, X}$ are $\mathfrak{M}_{0}$ together with a number of components isomorphic to $\mathbb{P}^{3}=$ $\mathbb{P}\left(H^{0}\left(\mathcal{O}_{\mathbb{P}^{1}}(1) \otimes H^{0}(\mathbf{d})\right)\right.$, one for each $g_{d}^{1}$ on $C$. For $d>(g+2) / 2$, there are two components, $\mathfrak{M}_{0}$ and a smooth component of dimension $\rho+3=2 d-g+1$, which is essentially a $\mathbb{P}^{3}$-bundle over $G_{d}^{1}$.

Next we discuss the analytic structure of the moduli space in a neighborhood of a singular point in case $b=2$, in other words how the components meet. Recall from Proposition 1.3 that the obstruction map has an intrinsically defined quadratic term given by $\alpha \cup \partial \alpha$.

Lemma 5.6. Let $D_{0}$ be given by the section $\sigma_{0}=\sum_{i} \alpha_{i} \otimes \beta_{i} \in H^{0}(\mathbf{b}) \otimes$ $H^{0}(\mathbf{d})$, where the $\alpha_{i}$ and $\beta_{i}$ are linearly independent and $V=\operatorname{span}\left\{\alpha_{i}\right\}$ is a quotient of $H^{0}(\mathbf{d})^{*}$ with dual space $V^{*} \subseteq H^{0}(\mathbf{d})$. Suppose that $V^{*} \neq H^{0}(\mathbf{d})$ and that the map $\mu_{0}$ is injective for $\mathbf{d}$. Then cup product induces a surjective 
map

$$
\begin{aligned}
H^{0}\left(\mathcal{O}_{X}\left(D_{0}\right)\right) / \mathbb{C} \sigma_{0} \otimes & \partial\left(H^{0}\left(\mathcal{O}_{D_{0}}\left(D_{0}\right)\right) \rightarrow H^{1}\left(\mathcal{O}_{X}\left(D_{0}\right)\right) / \sigma_{0} H^{1}\left(\mathcal{O}_{X}\right)\right. \\
& \cong\left(H^{0}(\mathbf{b}) / V\right) \otimes H^{1}(\mathbf{d}) .
\end{aligned}
$$

More precisely, for every $\tau_{2} \notin V^{*} \subset H^{0}(\mathbf{d})$, the map

$$
\left(H^{0}(\mathbf{b}) \otimes \mathbb{C} \tau_{2}\right) \otimes \partial\left(H^{0}\left(\mathcal{O}_{D_{0}}\left(D_{0}\right)\right) \rightarrow\left(H^{0}(\mathbf{b}) / V\right) \otimes H^{1}(\mathbf{d})\right.
$$

is surjective.

Proof. The image of $\partial: H^{0}\left(\mathcal{O}_{D_{0}}\left(D_{0}\right)\right) \rightarrow H^{1}\left(\mathcal{O}_{X}\right) \cong H^{0}\left(\mathcal{O}_{\mathbb{P}^{1}}\right) \otimes H^{1}\left(\mathcal{O}_{C}\right)$ is the set of all $1 \otimes \xi$, where $\sum_{i} \alpha \otimes \xi \beta_{i}=0$. Since the $\alpha_{i}$ are linearly independent, this condition is equivalent to the condition that $\xi \beta_{i}=0$ for all $i$. Cup product of such a class $1 \otimes \xi$ with

$$
\tau_{1} \otimes \tau_{2} \in H^{0}\left(\mathcal{O}_{X}\left(D_{0}\right)\right) \cong H^{0}(\mathbf{b}) \otimes H^{0}(\mathbf{d})
$$

is $\tau_{1} \otimes\left(\tau_{2} \cdot \xi\right) \in H^{1}\left(\mathcal{O}_{X}\left(D_{0}\right)\right)$. For the projection of this map to

$$
H^{1}\left(\mathcal{O}_{X}\left(D_{0}\right)\right) / \sigma_{0} H^{1}\left(\mathcal{O}_{X}\right) \cong\left(H^{0}(\mathbf{b}) / V\right) \otimes H^{1}(\mathbf{d})
$$

to be surjective, it suffices that, setting

$$
\left(V^{*}\right)^{\perp}=\left\{\xi \in H^{1}\left(\mathcal{O}_{C}\right): \xi \cdot \beta_{i}=0 \text { for all } i\right\},
$$

the induced cup product map $H^{0}(\mathbf{d}) \otimes\left(V^{*}\right)^{\perp} \rightarrow H^{1}(\mathbf{d})$ is surjective. Now by assumption, the adjoint $\mu_{0}^{*}$ of the $\mu_{0}$ map is surjective, where

$$
\mu_{0}^{*}: H^{1}\left(\mathcal{O}_{C}\right) \rightarrow H^{1}(\mathbf{d}) \otimes H^{0}(\mathbf{d})^{*}=\operatorname{Hom}\left(H^{0}(\mathbf{d}), H^{1}(\mathbf{d})\right) .
$$

Since $H^{0}(\mathbf{d}) \neq V$, there exists a $\tau_{2} \notin V$. Given $\eta \in H^{1}(\mathbf{d})$, there exists a linear map $F: H^{0}(\mathbf{d}) \rightarrow H^{1}(\mathbf{d})$ such that $F(V)=0$ and $F\left(\tau_{2}\right)=\eta$. The surjectivity of the map $\mu_{0}^{*}$ implies that $F$ is given by taking cup product with a $\xi$ such that $\xi \cdot \beta_{i}=0$ for all $i$ and $\xi \cdot \tau_{2}=\eta$. Thus the image of $H^{0}\left(\mathcal{O}_{X}\left(D_{0}\right)\right) / \mathbb{C} \sigma_{0} \otimes \partial\left(H^{0}\left(\mathcal{O}_{D_{0}}\left(D_{0}\right)\right)\right.$ contains every element of the form $\tau_{1} \otimes \eta$, where $\tau_{1}$ and $\eta$ are arbitrary, and so is all of $\left(H^{0}(\mathbf{b}) / V\right) \otimes H^{1}(\mathbf{d})$.

Using Lemma 5.6, we can describe the local structure of $H_{D_{0}, X}$ in case $b=2$ near a reducible divisor $\pi_{1}^{*} \mathbf{b}+\pi_{2}^{*} \mathbf{d}$, provided that $\mathbf{d}$ is generic in the sense that $h^{0}(\mathbf{d})$ is exactly 2 . 
Corollary 5.7. With assumptions on the $\mu_{0}$ map as above, suppose that $b=2$ and that $D_{0}=\pi_{1}^{*} \mathbf{b}+\pi_{2}^{*} \mathbf{d}$, where $h^{0}(\mathbf{d})=2$. Then an analytic neighborhood of $H_{D_{0}, X}$ near $D_{0}$ is biholomorphic to a neighborhood of the origin in $L_{0} \cup L_{1} \subset \mathbb{C}^{d+2}$, where $L_{0}$ is a hyperplane and $L_{1}$ is a linear space of dimension $2 d-g+1$, not contained in $L_{0}$.

Proof. The dimension of the Zariski tangent space $T$ to $H_{D_{0}, X}$ is $2 \cdot 2-$ $1+d-1=d+2$. The dimension of $\mathbb{P}^{1} \times C_{d}$ is $d+1$, and so the image $L_{0}$ of the tangent space to $\mathbb{P}^{1} \times C_{d}$ at $D_{0}$ has the expected dimension of a hyperplane in $T$. In fact, we have seen in the discussion prior to Lemma 5.2 that $L_{0}$ is indeed a hyperplane, defined by the linear form $\ell$, say. Thus if $\Phi: T \rightarrow \mathbb{C}^{g-d+1}$ is the Kuranishi obstruction map, defined in a neighborhood of the origin, then there exists a holomorphic function $f$ with differential $\ell$ such that $\Phi=f \Psi$, and the quadratic term in $\Phi$ is equal to $\ell \cdot d \Psi_{0}$. The span of $\alpha \cup \partial \alpha$ over all $\alpha$ is thus contained in the image of $d \Psi_{0}$ and this span is the same, after polarizing, as the image of $\alpha \cup \partial \beta+\beta \cup \partial \alpha$ over all $\alpha, \beta$. Using Lemma 5.6, there exists a choice of $\alpha_{i}, \beta_{i}$ with $\partial \alpha_{i}=0$ such that the obstruction space is generated by $\alpha_{i} \cup \partial \beta_{i}$. Thus $d \Psi_{0}$ has the same image as the map of Lemma 5.6 and so is surjective. It follows that $\Phi^{-1}(0)=$ $L_{0} \cup \Psi^{-1}(0)$, where $\Psi^{-1}(0)$ is a smooth submanifold of $T$ of codimension $g-d+1$. If it does not meet $L_{0}$ transversally, then $\operatorname{Ker} d \Psi_{0} \subseteq L_{0}$. But $L_{0} \cap H^{0}\left(\mathcal{O}_{X}\left(D_{0}\right)\right) / \mathbb{C} \sigma_{0}$ is the tangent space to the Segre embedding of $\mathbb{P}^{1} \times \mathbb{P}^{1}$ in $\mathbb{P} H^{0}\left(\mathcal{O}_{X}\left(D_{0}\right)\right)=\mathbb{P}^{3}$. Thus $H^{0}\left(\mathcal{O}_{X}\left(D_{0}\right)\right) / \mathbb{C} \sigma_{0}$ is not contained in $L_{0}$. On the other hand, $H^{0}\left(\mathcal{O}_{X}\left(D_{0}\right)\right) / \mathbb{C} \sigma_{0}$ is the tangent space to $\mathbb{P} H^{0}\left(\mathcal{O}_{X}\left(D_{0}\right)\right)$ and so is unobstructed, so that it must be contained in Ker $d \Psi_{0}$. Thus Ker $d \Psi_{0}$ is not contained in $L_{0}$, and so $\Psi^{-1}(0)$ meets $L_{0}$ transversally. This concludes the proof.

Finally, for a generic curve $C$, we shall use the description of the components of the moduli space above to make some calculations in case $b=2$ and $d$ is small. We do not need the description of the analytic structure of the moduli space. In this case the moduli space always has the component $\mathfrak{M}_{0}=\mathbb{P}^{1} \times \operatorname{Sym}^{d} C$. For $d=g-1 / 2, g / 2, g+1 / 2, \mathfrak{M}_{0}$ is the unique component, whereas in general the moduli space is equal to $\mathfrak{M}_{0} \cup \mathfrak{M}_{1}$. For $d=g+2 / 2, \mathfrak{M}_{1}$ is a union of $k$ copies of $\mathbb{P}^{3}$, where $k$ is the number of $g_{d}^{1}$ 's on $C$, and for $d>g+2 / 2 \mathfrak{M}_{1}$ is irreducible and smooth, of the expected dimension $\rho+3=2 d-g+1$.

To calulate the value of the Seiberg-Witten invariant, we shall first calculate the contribution from $\mathfrak{M}_{0}$ and then the contribution from $\mathfrak{M}_{1}$. Note 
that $\mathfrak{M}_{0}$ does not have the expected dimension, which is $2 d-g+1$. Moreover the moduli space is in general singular. However it is easy to see that we can choose incidence divisors $\mu_{1}, \ldots, \mu_{2 d-g+1}$ which meet properly in the smooth part of the moduli space. Following the procedure of Section 3 (see the comments at the end of the section), we first calculate the top Chern class of the obstruction bundle over $\mathfrak{M}_{0}$, which we have seen (Lemma 5.4) is the bundle $p_{1}^{*} T_{\mathbb{P}^{1}} \times p_{2}^{*} \mathcal{E}$, at least after cutting down by $\mu^{2 d-g+1}$. Here the $p_{i}$ are the projections of $\mathbb{P}^{1} \times \operatorname{Sym}^{d} C$ to the $i^{\text {th }}$ factor and $\mathcal{E}$ is the bundle $R^{1} \psi_{2 *} \mathcal{O}_{C \times C_{d}}(\mathcal{I})$, of rank $g-d$. The top Chern class of the tensor product of $p_{2}^{*} \mathcal{E}$ with the line bundle $p_{1}^{*} T_{\mathbb{P}^{1}}$ is: $c_{N}\left(p_{1}^{*} T_{\mathbb{P}^{1}} \times p_{2}^{*} \mathcal{E}\right)=\sum_{i=0}^{N} p_{1}^{*} c_{1}\left(T_{\mathbb{P}^{1}}\right)^{i} p_{2}^{*} c_{N-i}(\mathcal{E})$. If $h$ is the hyperplane class on $\mathbb{P}^{1}$, in other words the class of a point, then $p_{1}^{*} c_{1}\left(T_{\mathbb{P}^{1}}\right)=2 p_{1}^{*} h$ and $p_{1}^{*} c_{1}\left(T_{\mathbb{P}^{1}}\right)^{i}=0$ for $i>1$. Thus

$$
c_{N}\left(p_{1}^{*} T_{\mathbb{P}^{1}} \times p_{2}^{*} \mathcal{E}\right)=p_{2}^{*} c_{N}(\mathcal{E})+2 p_{1}^{*} h p_{2}^{*} c_{N-1}(\mathcal{E}) .
$$

By [1], p. 322, $c(\mathcal{E})=c\left(T_{C_{d}}\right)^{-1}=(1+x)^{g-1-d} e^{\theta / 1+x}$, where $x$ is the class of the divisor $C_{d-1} \subset C_{d}$ and $\theta$ is the pullback of the theta divisor on $\operatorname{Pic}^{d} C$ under the natural map, and moreover $\theta^{k} x^{d-k}=\frac{g !}{(g-k) !}$. To calculate the Seiberg-Witten invariant, we take $c_{N}\left(p_{1}^{*} T_{\mathbb{P} 1} \times p_{2}^{*} \mathcal{E}\right)$, where $N=g-d=1-a$. This gives a class in $H^{2 N}\left(\mathbb{P}^{1} \times \operatorname{Sym}^{d} C\right)$, and then we further multiply by $\mu^{d+1-N}$ and evaluate over the fundamental class. On $\mathbb{P}^{1} \times \operatorname{Sym}^{d} C$, it is clear that $\mu=p_{1}^{*} h+p_{2}^{*} x$, since $(t, p) \in \pi_{1}^{*}\{s\}+\pi_{2}^{*} \mathbf{d}$ if and only if either $t=s$ or $p \in \mathbf{d}$, and it is easy to see that the multiplicity is one. Thus we must calculate

$$
\begin{aligned}
& \left.\left(p_{1}^{*} h+p_{2}^{*} x\right)^{d+1-N} p_{2}^{*} c_{N}(\mathcal{E})+2 p_{1}^{*} h p_{2}^{*} c_{N-1}(\mathcal{E})\right) \\
& \quad=\left(2 p_{1}^{*} h\right) p_{2}^{*}\left(c_{N-1}(\mathcal{E}) x^{d+1-N}+(d+1-N) p_{1}^{*} h p_{2}^{*}\left(c_{N}(\mathcal{E}) x^{d-N}\right.\right. \\
& \quad=2 c_{N-1}(\mathcal{E}) x^{d+1-N}+(d+1-N) c_{N}(\mathcal{E}) x^{d-N}
\end{aligned}
$$

Plugging in for $c(\mathcal{E})$, we have

$$
\begin{aligned}
c(\mathcal{E}) & =(1+x)^{g-1-d} e^{\theta / 1+x}=(1+x)^{N-1} e^{\theta / 1+x} \\
& =\sum_{i=0}^{N-1}\left(\begin{array}{c}
N-1 \\
i
\end{array}\right) x^{i} \sum_{j=0}^{\infty} \frac{1}{j !} \theta^{j} \sum_{k=0}^{\infty}\left(\begin{array}{c}
-j \\
k
\end{array}\right) x^{k} .
\end{aligned}
$$


Thus for example the term involving $c_{N-1}(\mathcal{E})$ becomes

$$
\begin{aligned}
& 2 \sum_{i+j+k=N-1}\left(\begin{array}{c}
N-1 \\
i
\end{array}\right) \frac{1}{j !}\left(\begin{array}{c}
-j \\
k
\end{array}\right) x^{i+k+d+1-N} \theta^{j} \\
= & 2 \sum_{i+j+k=N-1}\left(\begin{array}{c}
N-1 \\
i
\end{array}\right) \frac{1}{j !}\left(\begin{array}{c}
-j \\
k
\end{array}\right) x^{d-j} \theta^{j} \\
= & 2 \sum_{i+j+k=N-1}\left(\begin{array}{c}
N-1 \\
i
\end{array}\right)\left(\begin{array}{c}
-j \\
k
\end{array}\right) \frac{1}{j !} \frac{g !}{(g-j) !} \\
= & 2 \sum_{j=0}^{N-1}\left(\sum_{k=0}^{N-1-j}\left(\begin{array}{c}
N-1 \\
N-1-j-k
\end{array}\right)\left(\begin{array}{c}
-j \\
k
\end{array}\right)\right)\left(\begin{array}{c}
N+d \\
j
\end{array}\right),
\end{aligned}
$$

where we have used $g=N+d$.

Applying Lemma 4.5 with $e=0$ to the inner sum above, where we let $a=N-1-j$ for a fixed $j$, we see that the expression reduces to $2 \sum_{j=0}^{N-1}\left(\begin{array}{c}N+d \\ j\end{array}\right)$

A very similar manipulation with the term $(d+1-N) c_{N}(\mathcal{E}) x^{d-N}$ gives

$$
(d+1-N) c_{N}(\mathcal{E}) x^{d-N}=(d+1-N)\left(\begin{array}{c}
N+d \\
N
\end{array}\right) .
$$

The final contribution for the component $\mathfrak{M}_{0}$ is therefore

$$
2 \sum_{j=0}^{N-1}\left(\begin{array}{c}
N+d \\
j
\end{array}\right)+(d+1-N)\left(\begin{array}{c}
N+d \\
N
\end{array}\right) .
$$

Note that $\mathfrak{M}_{0}$ is the unique component for $N=d+1, d, d-1$ corresponding to the cases $g=2 d+1,2 d, 2 d-1$. Plugging in, we find that the value of the invariant in case $N=d+1$ is

$$
2 \sum_{j=0}^{d}\left(\begin{array}{c}
2 d+1 \\
j
\end{array}\right)=\sum_{j=0}^{2 d+1}\left(\begin{array}{c}
2 d+1 \\
j
\end{array}\right)=(1+1)^{2 d+1}=2^{g} .
$$

Similar calculations handle the cases $N=d, d-1$, and again give the value $2^{g}$.

For $d=g+2 / 2, N=d-2, g=2 d-2$, the set $\mathfrak{M}_{1}$ consists of $k$ copies of $\mathbb{P}^{3}$, where $k$ is the number of $g_{d}^{1}$ 's on $C$. This number has been computed by Castelnuovo [1] p. 211: it is

$$
g ! \frac{1}{(g-d+1) !} \frac{1}{(g-d+2) !}=\frac{(2 d-2) !}{(d-1) ! d !} .
$$


In this case, the restriction of $\mathcal{D}$ to each piece $X \times \mathbb{P}^{3}$ is the incidence divisor, so that $\mu$ restricts to the hyperplane class in each $\mathbb{P}^{3}$. Thus the final answer: is

$$
2 \sum_{j=0}^{d-3}\left(\begin{array}{c}
2 d-2 \\
j
\end{array}\right)+3\left(\begin{array}{c}
2 d-2 \\
d-2
\end{array}\right)+\frac{(2 d-2) !}{(d-1) ! d !}
$$

which after a brief manipulation becomes $\sum_{j=0}^{2 d-2}\left(\begin{array}{c}2 d-2 \\ j\end{array}\right)=(1+1)^{2 d-2}=2^{g}$.

Somewhat more involved methods handle the case $N=d-3$, and presumably might be pushed, using excess intersections, to give the general. case. However, we shall give a simpler method for the calculation in the next section.

\section{Deformation to more general ruled surfaces.}

In this section, we shall study Seiberg-Witten moduli spaces, or equivalently the Hilbert scheme, for more general ruled surfaces $\mathbb{P}(V)$, where $V$ is a general (and in particular stable) rank two bundle over $C$. We shall deal with the case where $\operatorname{det} V$ has even degree, and thus assume that $c_{1}(V)=0$. Also, we shall only discuss the case of sections of $V$. However, it will be clear that our methods generalize to handle the case of odd degree as well as more general cases of multisections, and thus suffice for the homological calculations of the invariants in general. We will outline this approach at the end. Throughout this section, we fix a smooth curve $C$ of genus $g \geq 2$. it will not be necessary to assume that $C$ is generic in the Brill-Noether sense.

Recall that there is a one-to-one correspondence between irreducible sections $D_{0}$ of $\mathbb{P}(V)$ and line bundles $\lambda$ such that $V \otimes \lambda$ has a nowhere vanishing section, as follows: given a section $D_{0}$ of $\mathbb{P}(V)$, apply $R^{i} \pi_{*}$ to the exact sequence

$$
0 \rightarrow \mathcal{O}_{X} \rightarrow \mathcal{O}_{X}\left(D_{0}\right) \rightarrow \mathcal{O}_{D_{0}}\left(D_{0}\right) \rightarrow 0
$$

to obtain the exact sequence

$$
0 \rightarrow \mathcal{O}_{C} \rightarrow R^{0} \pi_{*} \mathcal{O}_{X}\left(D_{0}\right) \rightarrow \mathcal{O}_{D_{0}}\left(D_{0}\right) \rightarrow 0
$$

Here $R^{1} \pi_{*} \mathcal{O}_{X}=0$ since the fibers are $\mathbb{P}^{1}$ and we can write $R^{0} \pi_{*} \mathcal{O}_{X}\left(D_{0}\right)=$ $V^{\vee} \otimes \lambda=V \otimes \lambda$. Note that the normal bundle $\mathcal{O}_{D_{0}}\left(D_{0}\right)$ is naturally $\lambda^{2}$. The inverse map sends the section of $V^{\vee} \otimes \lambda$ to the homogeneous degree one subvariety of $\mathbb{P}(V)$ that it defines. 
If $D_{0}$ is not irreducible, then $D_{0}=E_{0}+\pi^{*}$ e. In this case the map $\mathcal{O}_{X} \rightarrow$ $\mathcal{O}_{X}\left(D_{0}\right)$ factors through $\mathcal{O}_{X}\left(E_{0}\right)$ and the induced map $\mathcal{O}_{C} \rightarrow R^{0} \pi_{*} \mathcal{O}_{X}\left(D_{0}\right)$ factors as

$$
\mathcal{O}_{C} \rightarrow R^{0} \pi_{*} \mathcal{O}_{X}\left(E_{0}\right) \rightarrow R^{0} \pi_{*} \mathcal{O}_{X}\left(E_{0}\right) \otimes \mathcal{O}_{C}(\mathbf{e})=R^{0} \pi_{*} \mathcal{O}_{X}\left(D_{0}\right) .
$$

Thus $D_{0}$ still corresponds to a section of $V^{\vee} \otimes \lambda$ for an appropriate $\lambda$, but the section vanishes exactly along e. Again such a section defines a subvariety of $\mathbb{P}(V)$, which is exactly $D_{0}$ since the section vanishes along $\mathbf{e}$. In this way, we can identify $\left|D_{0}\right|$ with $\mathbb{P} H^{0}\left(V^{\vee} \otimes \lambda\right)$, including the reducible fibers.

Proposition 6.1. Let e be an positive integer. For every line bundle $\lambda$ on $C$ of degree e,

(i) There exist stable bundles $V$ on $C$ together with an exact sequence

$$
0 \rightarrow \lambda^{-1} \rightarrow V \rightarrow \lambda \rightarrow 0 .
$$

(ii) For e $<(g-1) / 2$, and $V$ a generic stable bundle satisfying (i), if $\mu$ is a line bundle of degree $d \leq e$ and $H^{0}(V \otimes \mu) \neq 0$, then $\mu=\lambda$ and $H^{0}(V \otimes \lambda)$ has dimension one.

(iii) For $e=(g-1) / 2$ and $V$ general, there are exactly $2^{g}$ distinct $\lambda$ with $H^{0}(V \otimes \lambda) \neq 0$, and for each such $\lambda$, $\operatorname{dim} H^{0}(V \otimes \lambda)=1$. Moreover, if $\operatorname{deg} \mu<(g-1) / 2$, then $H^{0}(V \otimes \mu)=0$.

Proof. For a line bundle $\lambda$ of degree $e>0, \operatorname{Ext}^{1}\left(\lambda, \lambda^{-1}\right)=H^{1}\left(\lambda^{-2}\right)$ which has dimension $2 e+g-1$, by Riemann-Roch. Let $V$ be a rank two bundle corresponding to an extension class $\xi \in H^{1}\left(\lambda^{-2}\right)$. Suppose that there exists a nonzero map $\mu \rightarrow V$, where $\operatorname{deg} \mu=d \leq e$, and that $\mu \neq \lambda$ in case $d=e$. Since $H^{0}\left(\mu \otimes \lambda^{-1}\right)=0$ since $\mu \otimes \lambda^{-1}$ either has negative degree or has degree zero and is not trivial, there must exist a nonzero section of $\mu \otimes \lambda$ which lifts to a section of $\mu \otimes V$. Note that (s), the divisor of zeroes of $s$, has degree $d+e$, and we can identify the set of all pairs $(\mu, s)$ such that $\mu$ is a line bundle of degree $d$ and $s$ is a nonzero section of $\mu \otimes \lambda$, mod scalars, with $C_{d+e}$.

The section $s$ lifts to a section of $\mu \otimes V$ if and only if the coboundary map $\partial(s)=0$, where $\partial s \in H^{1}\left(\mu \otimes \lambda^{-1}\right)$. Now $\partial s=\xi \cdot s$, the cup product of $s \in H^{0}(\mu \otimes \lambda)$ with $\xi \in H^{1}\left(\lambda^{-2}\right)$. Consider the exact sequence

$$
0 \rightarrow \lambda^{-2} \stackrel{\times s}{\longrightarrow} \lambda^{-1} \otimes \mu \rightarrow \mathcal{O}_{\mathbf{f}} \rightarrow 0
$$


where $\mathbf{f}=(s) \in C_{d+e}$. By assumption, $\partial s=0$ if and only if $\xi \cdot s=0$ if and only if the image of $\xi$ in $H^{1}\left(\lambda^{-1} \otimes \mu\right)$ is zero, if and only if $\xi$ is in the image of $H^{0}\left(\mathcal{O}_{\mathbf{f}}\right)$. By assumption either $\operatorname{deg}\left(\lambda^{-1} \otimes \mu\right)<0$ or $\operatorname{deg}\left(\lambda^{-1} \otimes \mu\right)=0$ but $\lambda^{-1} \otimes \mu$ is not trivial. In either case $H^{0}\left(\lambda^{-1} \otimes \mu\right)=0$, and so the image of $H^{0}\left(\mathcal{O}_{\mathrm{f}}\right)$ has dimension $d+e$. Thus the set of possible extension classes $\xi$ for which a given $s$ lifts has dimension $d+e$, and so corresponds to a $\mathbb{P}^{d+e-1} \subseteq \mathbb{P} H^{1}\left(\lambda^{-2}\right)=\mathbb{P}^{2 e+g-2}$. The set of all $\xi$ for which some $s$ lifts is then the union over all $s$ of a linear subspace of $\mathbb{P}^{2 e+g-2}$ of dimension $d+e-1$. Since the set of all $s$ is just $C_{d+e}$, the dimension of the set of all possible $\xi$ is at most $2 d+2 e-1$. This number is less than $2 e+g-2$ exactly when $d<(g-1) / 2$. Choosing a bundle $V$ coresponding to an extension class $\xi$ in the complement of this set gives a bundle $V$, written as an extension of $\lambda$ by $\lambda^{-1}$, such that, if $H^{0}(V \otimes \mu) \neq 0$ and $\operatorname{deg} \mu \leq \lambda$, then $\mu=\lambda$. In particular, $V$ is stable, proving (i) and (ii), except for the statement that $\operatorname{dim} H^{0}(V \otimes \lambda)=1$.

To see the statement about $\operatorname{dim} H^{0}(V \otimes \lambda)$, if $s$ is a nonzero section of $\lambda^{2}$ which lifts to a section of $V \otimes \lambda$, then arguments similar to those above show that the orthogonal complement of $s \cdot H^{0}\left(K_{C}\right)$ in $H^{1}\left(\lambda^{2}\right)$ has dimension $2 e-1$ and so gives a linear space of dimension $2 e-2$ inside $\mathbb{P}^{2 e+g-2}$. Moreover, the possible $s$ correspond to the case $\mu=\lambda$, and so form a proper subvariety of $C_{d+e}=C_{2 e}$. In all, the $\xi$ for which some $s$ lifts, such that the corresponding line bundle $\mu=\lambda$, form a subvariety of $\mathbb{P}^{2 e+g-2}$ of dimension at most $4 e-3$. Now $4 e-3<2 e+g-2$ provided that $e<(g+1) / 2$, and thus certainly if $e \leq(g-1) / 2$. This establishes the last statement of (ii).

The remaining assertion (iii) is a classical formula due to Corrado Segre [11]. It follows from our formulas in the previous section for the zerodimensional invariant, and will be reproved in more generality shortly.

We now fix a bundle $V$ which will later be assumed generic in an appropriate sense. For each $d$, we consider the following varieties of Brill-Noether type:

$$
\begin{aligned}
& W_{1, d}(V)=\left\{\lambda \in \operatorname{Pic}^{d} C: h^{0}(V \otimes \lambda) \geq 1\right\} \\
& G_{1, d}(V)=\left\{(s, \lambda): \lambda \in \operatorname{Pic}^{d} C, s \in \mathbb{P}\left(H^{0}(V \otimes \lambda)\right)\right\} .
\end{aligned}
$$

Thus there is a natural map $G_{1, d}(V) \rightarrow W_{1, d}(V)$. It is also clear that, with $X=\mathbb{P}(V), G_{1, d}(V)$ is exactly the Hilbert scheme of $X$ corresponding to sections (possibly reducible) of the appropriate degree and that the map $G_{1, d}(V) \rightarrow W_{1, d}(V)$ can be identified with the map from the Hilbert scheme 
to Pic $X$. We wish to give another construction of the Hilbert scheme in this context; in other words, we will put another scheme structure on $G_{1, d}(V)$ and then claim that it is in fact the usual one. To do so, we make a construction similar to the usual construction of Brill-Noether theory: fix a divisor $D$ on $C$ of degree $m \gg 0$ such that $h^{1}\left(V \otimes \lambda \otimes \mathcal{O}_{C}(D)\right)=0$ for all line bundles $\lambda$ of degree $d$. We can assume that $D$ is an effective divisor consisting of reduced points of $C$ if we wish. Consider the restriction sequence

$$
0 \rightarrow V \otimes \lambda \rightarrow V \otimes \lambda \otimes \mathcal{O}_{C}(D) \rightarrow V \otimes \lambda \otimes \mathcal{O}_{D}(D) \rightarrow 0 .
$$

Taking global sections, there is a map

$$
\varphi: H^{0}\left(V \otimes \lambda \otimes \mathcal{O}_{C}(D)\right) \rightarrow H^{0}\left(V \otimes \lambda \otimes \mathcal{O}_{D}(D)\right) .
$$

The first vector space has dimension $2 m+2 d-2 g+2$, by Riemann-Roch, and the second has dimension $2 m$, and $\lambda \in W_{1, d}(V)$ if and only if $\varphi$ has a kernel. In this case, the fiber over $\lambda$ in $G_{1, d}(V)$ is just $\mathbb{P}(\operatorname{Ker} \varphi)=\mathbb{P}\left(H^{0}(V \otimes \lambda)\right)$. Globally, let $\mathcal{P}$ be a Poincaré line bundle for $C \times \operatorname{Pic}^{d} C$, and let $\pi_{i}$ be the projection of $C \times \operatorname{Pic}^{d} C$ to the $i^{\text {th }}$ factor. Set

$$
\begin{aligned}
\mathcal{E}^{\prime} & =\pi_{2 *}\left(\mathcal{P} \otimes \pi_{1}^{*}\left(V \otimes \mathcal{O}_{C}(D)\right)\right) ; \\
\mathcal{E}^{\prime \prime} & =\pi_{2 *}\left(\mathcal{P} \otimes \pi_{1}^{*}\left(V \otimes \mathcal{O}_{D}(D)\right)\right),
\end{aligned}
$$

so that there is a natural evaluation map $\Phi: \mathcal{E}^{\prime} \rightarrow \mathcal{E}^{\prime \prime}$. Then $W_{1, d}(V)$ is the scheme where $\Phi$ fails to be injective. We can define $G_{1, d}(V)$ similarly: let $p: \mathbb{P} \mathcal{E}^{\prime} \rightarrow \mathrm{Pic}^{d} C$ be the projection. We have the inclusion of $\mathcal{O}_{\mathbb{P} \mathcal{E}^{\prime}}(-1)$ inside $p^{*} \mathcal{E}^{\prime}$. Consider the composition

$$
\mathcal{O}_{\mathbb{P} \mathcal{E}^{\prime}}(-1) \rightarrow p^{*} \mathcal{E}^{\prime} \stackrel{p^{*} \Phi}{\longrightarrow} p^{*} \mathcal{E}^{\prime \prime}
$$

If we denote this composition by $\tilde{\Phi}$, then $\tilde{\Phi}=0$ at a point $(s, \lambda)$, where $\lambda \in \operatorname{Pic}^{d} C$ and $s \in \mathbb{P} H^{0}\left(V \otimes \lambda \otimes \mathcal{O}_{C}(D)\right)$, if and only if $s$ is in the image of $\mathbb{P} H^{0}(V \otimes \lambda)$. Thus the vanishing of $\tilde{\Phi}$ defines $G_{1, d}(V)$ as a set inside $\mathbb{P} \mathcal{E}^{\prime}$. Note that $\mathbb{P} \mathcal{E}^{\prime}$ is itself a Hilbert scheme: it is the same as $G_{1, d+m}(V)$, corresponding to the set of all sections of $X$ of degree $2 d+2 m$. Moreover $G_{1, d}(V)$ is the subset of $\mathbb{P} \mathcal{E}^{\prime}$ consisting exactly of those sections containing $\pi^{*} D$. We leave it to the reader to work through the details that the subscheme defined by $\tilde{\Phi}$ represents the functor corresponding to $G_{1, d}(V)$ (see [1] pp. 182-184 for the Brill-Noether analogue) and that indeed this identifies $G_{1, d}(V)$ with the Hilbert scheme as schemes.

Next suppose that $(s, \lambda)$ is a point of $G_{1, d}(V)$ such that the section $s$ does not vanish. Standard arguments (cf. [1], pp. 185-186) identify 
the Zariski tangent space to $G_{1, d}(V)$ at the point $(s, \lambda)$ with $H^{0}\left(\lambda^{2}\right)=$ $\operatorname{Hom}\left(\lambda^{-1}, V / \lambda^{-1}\right)$, via the exact sequence

$$
0 \rightarrow H^{0}\left(\mathcal{O}_{C}\right) \rightarrow H^{0}(V \otimes \lambda) \rightarrow H^{0}\left(\lambda^{2}\right) \rightarrow H^{1}\left(\mathcal{O}_{C}\right) \rightarrow H^{1}(V \otimes \lambda) .
$$

Moreover the differential of the map from $G_{1, d}(V)$ to $W_{1, d}(V)$ is the obvious map $H^{0}\left(\lambda^{2}\right) \rightarrow H^{1}\left(\mathcal{O}_{C}\right)$. Finally, a standard cocycle calculation identifies the obstruction space as $H^{1}\left(\lambda^{2}\right)$. Note that, if $\lambda$ corresponds to the section $D_{0}$ of $X$, then $H^{1}\left(\lambda^{2}\right)=H^{1}\left(\mathcal{O}_{D_{0}}\left(D_{0}\right)\right)$ is the same obstruction we would have found via the Hilbert scheme, as well it must be since $G_{1, d}(V)$ represents the same functor as the Hilbert scheme.

For a general section $s$, suppose that the map $\mathcal{O}_{C} \rightarrow V \otimes \lambda$ vanishes along the divisor $\mathbf{e}$, so that there is a factorization

$$
\mathcal{O}_{C} \rightarrow V \otimes \lambda \otimes \mathcal{O}_{C}(-\mathbf{e}) \rightarrow V \otimes \lambda .
$$

Let $\lambda_{0}=\lambda \otimes \mathcal{O}_{C}(-\mathbf{e})$. Then there is a commutative diagram

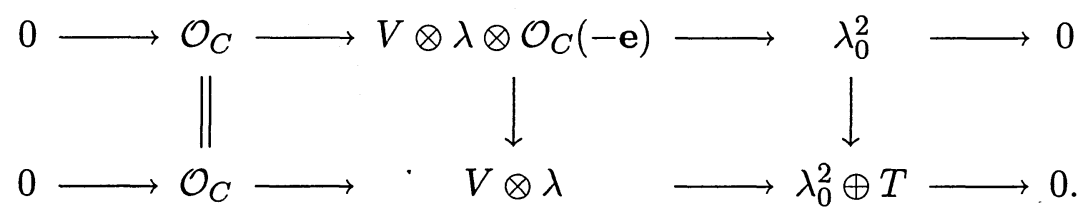

Here $T$ is a skyscraper sheaf isomorphic to $V \otimes \mathcal{O}_{\mathbf{e}}$, and so has length $2 \operatorname{deg} \mathbf{e}=2 e$, say. We can again identify the Zariski tangent space to $G_{1, d}(V)$ at $(s, \lambda)$ with

$\operatorname{Hom}\left(\lambda^{-1}, V / \lambda^{-1}\right)=H^{0}\left(V \otimes \lambda / s \cdot \mathcal{O}_{C}\right)=H^{0}\left(\lambda_{0}^{2} \oplus T\right)=H^{0}\left(\lambda_{0}^{2}\right) \oplus H^{0}(T)$.

Moreover the obstruction space is

$$
\operatorname{Ext}^{1}\left(\lambda^{-1}, V / \lambda^{-1}\right)=H^{1}\left(\lambda_{0}^{2} \oplus T\right)=H^{1}\left(\lambda_{0}^{2}\right) .
$$

This again corresponds to the deformation theory and obstruction theory for the Hilbert scheme: let $D_{0}=E_{0}+\pi^{*} \mathbf{e}$, where $E_{0}$ is an irreducible section of $X$. Apply $R^{i} \pi_{*}$ to the exact sequence

$$
0 \rightarrow \mathcal{O}_{X} \rightarrow \mathcal{O}_{X}\left(D_{0}\right) \rightarrow \mathcal{O}_{D_{0}}\left(D_{0}\right) \rightarrow 0
$$

using $R^{1} \pi_{*} \mathcal{O}_{X}=R^{1} \pi_{*} \mathcal{O}_{X}\left(D_{0}\right)=0$. We obtain

$$
0 \rightarrow \mathcal{O}_{C} \rightarrow V \otimes \lambda \rightarrow \pi_{*} \mathcal{O}_{D_{0}}\left(D_{0}\right) \rightarrow 0,
$$

and $R^{1} \pi_{*} \mathcal{O}_{D_{0}}\left(D_{0}\right)=0$. Thus

$$
H^{i}\left(\mathcal{O}_{D_{0}}\left(D_{0}\right)\right)=H^{i}\left(\pi_{*} \mathcal{O}_{D_{0}}\left(D_{0}\right)\right)=H^{i}\left(V \otimes \lambda / s \cdot \mathcal{O}_{C}\right) .
$$

Summarizing, we have shown the following: 
Proposition 6.2. Let $(s, \lambda)$ be a point of $G_{1, d}(V)$. Suppose that the section $s$ vanishes exactly along the effective divisor $\mathbf{e}$, and set $\lambda_{0}=\lambda \otimes \mathcal{O}_{C}(-\mathbf{e})$. Then the Zariski tangent space to $G_{1, d}(V)$ is $H^{0}\left(V \otimes \lambda / s \cdot \mathcal{O}_{C}\right)$, which has dimension $h^{0}\left(\lambda_{0}^{2}\right)+2 e$, and the obstruction space is $H^{1}\left(\lambda_{0}^{2}\right)$.

Assuming for simplicity that $s$ does not vanish at any point, and so $\lambda=\lambda_{0}$ in the above notation, the group $H^{1}\left(\lambda^{2}\right)$ arises in yet another way as follows: the universal extension over $\mathbb{P} H^{1}\left(\lambda^{2}\right)$ of $\lambda$ by $\lambda^{-1}$ gives rise to a Kodaira-Spencer map from the tangent space of $\mathbb{P} H^{1}\left(\lambda^{-2}\right)$ at a nonzero point $\xi \in H^{1}\left(\lambda^{-2}\right)$, namely $H^{1}\left(\lambda^{-2}\right) / \mathbb{C} \cdot \xi$, to $H^{1}$ (ad $\left.V\right)$. A diagram chase identifies the cokernel of this map with $H^{1}\left(\lambda^{2}\right)$ under the natural map $H^{1}(\operatorname{ad} V) \rightarrow H^{1}\left(\lambda^{2}\right)$. Thus $H^{1}\left(\lambda^{2}\right)=0$ if and only if the map from the set of extensions to moduli is a submersion at $\xi$. If $d \geq(g-1) / 2$, then for a generic choice of $\lambda$ we will indeed have $H^{1}\left(\lambda^{2}\right)=0$, and so the map from extensions to moduli will be a submersion where defined.

We now show that, for a generic choice of $V$, the Hilbert scheme is always smooth:

Proposition 6.3. For a generic stable bundle $V$ with $c_{1}(V)=0$ and for all $d$, the Hilbert scheme of sections of $X=\mathbb{P}(V)$ of square $2 d$ is everywhere smooth of the expected dimension $2 d-g+1$.

Proof. Fix a value for $d$ corresponding to the degree of a line subbundle $\lambda$ of $V$. We have seen that, for generic $V$, the space of sections is empty if $d<(g-1) / 2$, and if $\lambda$ has degree $>g-1$, then $h^{1}\left(\lambda^{2}\right)=0$ by Serre duality. For $(g-1) / 2 \leq d \leq g-1$, let $\mathcal{P}$ be the Poincaré line bundle over $C \times \operatorname{Pic}^{d} C$ and let $\mathcal{V}=R^{1} \pi_{2 *} \mathcal{P}^{\otimes-2}$. Note that, as $\operatorname{deg} \lambda=d>0$, then $h^{0}\left(\lambda^{\otimes-2}\right)=0$ and $h^{1}\left(\lambda^{\otimes-2}\right)=2 d+g-1$. Hence $\mathcal{V}$ is a vector bundle of rank $2 d+g-1$ over $\operatorname{Pic}^{d} C$ and $\mathbb{P V}$ is a $\mathbb{P}^{2 d+g-2}$-bundle over $\operatorname{Pic}^{d} C$. The space $\mathbb{P V}$ is a moduli space for vector bundles $V$ given as extensions. There is an open subset $\mathcal{U}$ of $\mathbb{P V}$ corresponding to stable bundles, which is nonempty by (i) of (6.1). The remarks prior to the statement of (6.3) imply that the morphism $\mathcal{U} \rightarrow \mathfrak{M}(C)$ is dominant, where $\mathfrak{M}(C)$ is the moduli space of stable rank two bundles $V$ over $C$ with $c_{1}(V)=0$. Let

$$
\mathcal{B}=\left\{\lambda \in \operatorname{Pic}^{d} C: h^{1}\left(\lambda^{2}\right) \neq 0\right\} .
$$

Since $h^{1}\left(\lambda^{2}\right) \neq 0$ if and only if $h^{0}\left(K_{C} \otimes \lambda^{-2}\right) \neq 0, \mathcal{B}$ is the inverse image in $\mathrm{Pic}^{d} C$ of the set of effective divisors in $\mathrm{Pic}^{2 g-2-2 d} C$ under the obvious 
(étale) map $\lambda \mapsto K_{C} \otimes \lambda^{-2}$. Thus $\mathcal{B}$ has the same dimension as the set of effective divisors in $\mathrm{Pic}^{2 g-2-2 d} C$, namely $2 g-2-2 d$. Hence if $p: \mathbb{P V} \rightarrow$ $\mathrm{Pic}^{d} C$ is the projection, then

$$
\operatorname{dim} p^{-1}(\mathcal{B})=2 g-2-2 d+2 d+g-2=3 g-4 .
$$

It follows that the image of $p^{-1}(\mathcal{B}) \cap \mathcal{U}$ in $\mathfrak{M}(C)$ cannot be all of $\mathfrak{M}(C)$. Thus we can choose a stable bundle $V$ such that, if $\operatorname{deg} \lambda<(g-1) / 2$, then there is no nonzero section of $V \otimes \lambda$, and if $\operatorname{deg} \lambda \geq(g-1) / 2$ and there is a nowhere vanishing section $s$ of $V \otimes \lambda$, then $h^{1}\left(\lambda^{2}\right)=0$. It follows that $(s, \lambda)$ is a smooth point of $G_{1, d}(V)$ (or of the Hilbert scheme), and the discussion prior to Proposition 6.2 shows how to extend this to all nonzero sections. Thus the Hilbert scheme of sections is everywhere smooth.

Next we turn to the enumerative geometry of the Hilbert scheme. Since $\mathcal{O}_{\mathbb{P E}^{\prime}}(-1)$ is a line bundle, $\tilde{\Phi}$ is equivalent to a section of $\mathcal{O}_{\mathbb{P} \mathcal{E}^{\prime}}(1) \otimes \mathcal{E}^{\prime \prime}$ and the class of its zero set, namely $G_{1, d}(V)$, is given by $c_{2 m}\left(\mathcal{O}_{\mathbb{P} \mathcal{E}^{\prime}}(1) \otimes \mathcal{E}^{\prime \prime}\right)$. If we set $\zeta=c_{1}\left(\mathcal{O}_{\mathbb{P}^{\prime}}(1)\right)$ and use the fact that $\mathcal{E}^{\prime \prime}$ is a topologically trivial bundle of rank $2 m$ [1] p. 309 , then $c_{2 m}\left(\mathcal{O}_{\mathbb{P E}}(1) \otimes \mathcal{E}^{\prime \prime}\right)$ is the term of degree $2 m$ in $(1+\zeta)^{2 m}$, namely $\zeta^{2 m}$. In particular, the class of $W_{1, d}(V)$ is given by $p_{*} \zeta^{2 m}$, which is the appropriate Segre class of $\mathcal{E}^{\prime}$. To find it, take $c\left(\mathcal{E}^{\prime}\right)^{-1}$. Topologically $\mathcal{E}^{\prime}$ is two copies of $\pi_{2 *}\left(\mathcal{P} \otimes \pi_{1}^{*} \mathcal{O}_{C}(D)\right)$, and using [1] p. 336, this last bundle has total Chern class $e^{-\theta}$. Thus $c\left(\mathcal{E}^{\prime}\right)=e^{-2 \theta}$ and $c\left(\mathcal{E}^{\prime}\right)^{-1}=$ $e^{2 \theta}$. Taking the term of degree $2 g-2 d-1$, we find [6]:

$$
\left[W_{1, d}(V)\right]=\frac{(2 \theta)^{2 g-2 d-1}}{(2 g-2 d-1) !} .
$$

In particular, when $2 d+1=g$ we obtain $(2 \theta)^{g} / g !=2^{g}$, giving the formula of Segre for the case of the zero-dimensional invariant. (In fact, Segre stated the formula for all values of $d$.)

To handle the general case, we use:

Proposition 6.4. The $\mu$ divisor on $G_{1, d}(V)$ is algebraically equivalent to the restriction of $\zeta=c_{1}\left(\mathcal{O}_{\mathbb{P} \mathcal{E}^{\prime}}(1)\right)$.

Corollary 6.5. The value of the Seiberg-Witten invariant is $2^{g}$.

Proof of the corollary. We need to compute $\zeta^{2 m+2 d-2 g+1}$. This is the top Segre class of $\mathcal{E}^{\prime}$, and by the calculations above it is equal to the degree $g$ term in $e^{2 \theta}$, namely $2^{g}$. 
Proof of (6.4). Keeping our previous notation, note that $\mathbb{P} \mathcal{E}^{\prime}$ is itself a Hilbert scheme, namely the scheme of all sections of $X$ of degree $2 d+2 m$, and the Hilbert scheme is the subscheme of all sections which are of the form $D_{0}+\pi^{*} D$. Thus, choosing a point $p \in X$ not lying in $\pi^{*} D$, the incidence divisor $\mu(p)$ for the Hilbert scheme is the restriction of the corresponding incidence divisor on $\mathbb{P} \mathcal{E}^{\prime}$. Recall that $\mathcal{E}^{\prime}=\pi_{2 *}\left(\mathcal{P} \otimes \pi_{1}^{*}\left(V \otimes \mathcal{O}_{C}(D)\right)\right)$. Let $t=\pi(p)$, and suppose that we have chosen the Poincaré line bundle $\mathcal{P}$ so that $\mathcal{P} \mid\{t\} \times \operatorname{Pic}^{d} C$ is trivial. Fix the line $\ell \subset V_{t}$ corresponding to $p \in X$. Identifying the space $\left(V_{t} / \ell\right) \otimes_{\mathbb{C}} \mathcal{O}_{C}(D)_{t}$ with $\mathbb{C}$, there is then a surjection

$$
\begin{aligned}
\mathcal{P} \otimes \pi_{1}^{*}\left(V \otimes \mathcal{O}_{C}(D)\right) & \rightarrow \mathcal{P} \otimes \pi_{1}^{*}\left(V \otimes \mathcal{O}_{C}(D)\right) \mid\{t\} \times \operatorname{Pic}^{d} C \rightarrow \\
& \rightarrow \mathcal{P} \mid\{t\} \times \operatorname{Pic}^{d} C \cong \mathcal{O}_{\mathrm{Pic}^{d} C} .
\end{aligned}
$$

Applying $\pi_{2 *}$, we get a map $F: \mathcal{E}^{\prime} \rightarrow \mathcal{O}_{\operatorname{Pic}^{d} C}$, which is nonzero if $D$ is sufficiently ample. Clearly $F(s, \lambda)=0$ exactly when $s(t) \in \ell \subset V_{t}$. In other words, the zero set of $F$ is the incidence divisor $\mathcal{D}$ corresponding to the point $p$. Now

$$
\begin{aligned}
F \in & \operatorname{Hom}\left(\mathcal{E}^{\prime}, \mathcal{O}_{\mathrm{Pic}^{d} C}\right)=H^{0}\left(\operatorname{Pic}^{d} C ;\left(\mathcal{E}^{\prime}\right)^{\vee}\right) \\
& =H^{0}\left(\operatorname{Pic}^{d} C ; p_{*} \mathcal{O}_{\mathbb{P} \mathcal{E}^{\prime}}(1)\right)=H^{0}\left(\mathbb{P E}^{\prime} ; \mathcal{O}_{\mathbb{P} \mathcal{E}^{\prime}}(1)\right) .
\end{aligned}
$$

Running through the identifications above, we see that the zero set of $F$ is exactly the zero set of the induced section of $\mathcal{O}_{\mathbb{P} \mathcal{E}^{\prime}}(1)$, and a straightforward argument also checks the multiplicity. Thus $\mathcal{D}$ is the zero set of a section of $\mathcal{O}_{\mathbb{P} \mathcal{E}^{\prime}}(1)$, and so $[\mathcal{D}]=\zeta$.

We can apply the methods above to handle enumerative questions not directly related to Seiberg-Witten theory. For example, there is the following calculation via the Grothendieck-Riemann-Roch theorem:

Theorem 6.6. Suppose that $g$ is even. Then the set of stable rank two bundles $V$ with $\operatorname{det} V=0$ such that there exists a line bundle $\lambda$ of degree $(g-2) / 2$ with $h^{0}(V \otimes \lambda) \neq 0$ is an irreducible divisor in the moduli space $\mathfrak{M}(C)$. Its class, at least as a divisor on the moduli functor, is $2^{g} \Delta$, where $\Delta$ is the first Chern class of the determinant line bundle, which again exists on the moduli functor.

Finally let us give the general formula for the Seiberg-Witten invariant:

Theorem 6.7. Let $X=\mathbb{P}^{1} \times C$ be a product ruled surface and let $L$ be a line bundle on $X$ of type $(2 a, 2 b)$, with $b \geq 1$ and $(1-g) / b \leq a<0$. Then 
for a Kähler metric $\omega$ such that $\omega \cdot L<0$, the value of the Seiberg-Witten invariant on $L$ is $b^{g}$.

Note that this formula is a special case of a general transition formula for Seiberg-Witten invariants, which has been established by $\mathrm{Li}$ and Liu [9] as well as the authors (unpublished). It would also follow, by copying the arguments above for the case of sections, but working with $\operatorname{Sym}^{m} V$ (for $m=b-1$ ) instead of $V$, if we knew that, for a general ruled surface, the Hilbert scheme was always smooth of the expected dimension. We state this as a conjecture:

Conjecture. Let $X$ be a general ruled surface. Then every component of the Hilbert scheme is smooth of the expected dimension. Here, since $H^{2}\left(\mathcal{O}_{X}\right)=0$, the expected dimension of the Hilbert scheme at a curve $D$ is $\frac{1}{2}\left(D^{2}-D \cdot K_{X}\right)$.

Without assuming this conjecture, one can deduce the result from the methods of Section 3 in case the Hilbert scheme is smooth but does not have the expected dimension, and with more work in general, again by reducing it to a homological calculation in a space along the lines of $\mathbb{P} \mathcal{E}^{\prime}$.

Note added in proof. Another and more general proof of the wallcrossing formula has been given by C. Okonek and A. Teleman, SeibergWitten invariants for manifolds with $b_{+}=1$ and the universal wall crossing formula, Internat. J. Math. 7 (1996), 811-832.

\section{References.}

[1] E. Arbarello, M. Cornalba, P.A. Griffiths and J. Harris, Geometry of Algebraic Curves volume I, Springer Verlag, New York Berlin Heidelberg Tokyo, 1985.

[2] R. Brussee, The canonical class and the $C^{\infty}$ properties of Kähler surfaces, (electronic) New York J. Math. 2 (1996), 103-146.

[3] R. Fintushel, P. Kronheimer, T. Mrowka, R. Stern, and C. Taubes, to appear.

[4] R. Friedman and J.W. Morgan, Smooth Four-Manifolds and Complex Surfaces, Ergebnisse der Mathematik und ihrer Grenzgebiete 3. Folge 27, Springer, Berlin Heidelberg New York, 1994.

[5] Algebraic surfaces and Seiberg-Witten invariants, J. Algebraic Geometry, 6 (1997), 445-479. 
Obstruction bundles, semiregularity, and Seiberg-Witten invariants 495

[6] F. Ghione, Quelques résultats de Corrado Segre sur les surfaces réglées, Math. Annalen, 255 (1981), 77-95.

[7] K. Kodaira and D.C. Spencer, A theorem of completeness of characteristic systems of complete continuous systems, Amer. J. Math. 81 (1959), 477-500.

[8] H. Lange, Höhere Sekantenvarietäten und Vektorbündel auf Kurven, Manuscripta Math. 52 (1985), 63-80.

[9] T.J. Li and A. Liu, General wall crossing formula, Math. Research Letters, 2 (1995), 797-810.

[10] D. Mumford, Lectures on Curves on an Algebraic Surface, Annals of Mathematics Studies 59, Princeton University Press, Princeton, NJ, 1966.

[11] C. Segre, Recherches générales sur les courbes et les surfaces réglées algébriques II, Math. Annalen, 34 (1889), 1-25.

[12] E. Witten, Monopoles and four-manifolds, Math. Research Letters, 1 (1994), 769-796.

Received October 18, 1995.

COLUMBIA UNIVERSITY, NEW YORK, NY 10027, USA

E-mail address: rf@math.columbia.edu, jm@math.columbia.edu 